\title{
Asymptotic Stability of the Relativistic Maxwellian
}

By

\author{
Robert T. GlasseY* and Walter A. Strauss**
}

\begin{abstract}
Solutions of the relativistic Boltzmann equation are studied for all initial data which are periodic in the space variable and near equilibrium. An equilibrium is a relativistic Maxwellian distribution of momenta. Under appropriate conditions on the scattering kernel, this equilibrium is asymptotically stable in a variety of function spaces.
\end{abstract}

\section{Introduction}

Although the classical (non-relativistic) Boltzmann equation has been heavily studied, the relativistic version has received scant attention. The relativistic Boltzmann euqation is

$$
V \cdot \nabla_{X} F=-C(F, F) .
$$

Here the dot represents the Lorentz inner product (+---) of 4-vectors, $v=\left(v_{1}, v_{2}, v_{3}\right), V=\left(v_{0}, v_{1}, v_{2}, v_{3}\right), X=\left(x_{0}, x_{1}, x_{2}, x_{3}\right), x=\left(x_{1}, x_{2}, x_{3}\right), x_{0}=-t$ and $C(F, F)$ is the collision integral. Normalizing the speed of light $c=1$ and the particle mass $m=1$, we have $V \cdot V=1$ or $v_{0}=\sqrt{1+|v|^{2}}$. For our purposes it is convenient to separate the time and space variables and to divide $(R B)_{0}$ by $v_{0}$ to obtain

$$
\partial_{t} F+\hat{v} \cdot \nabla_{x} F=Q(F, F)
$$

where $Q(F, F)=v_{0}^{-1} C(F, F)$ and

Communicated by H. Araki, July 22, 1992.

1991 Mathematics Subject Classification: 82 A 45

* Dept. of Mathematics, Indiana University, Bloomington, IN 47405, USA

** Dept. of Mathematics, Brown University, Providence, RI 02912, USA

Research supported in part by National Science Foundation grants DMS 90-23864 and DMS 90-23196 and by ARO grant DAAL-3-90-G0012. 


$$
\hat{v}=\frac{v}{v_{0}}=\frac{v}{\sqrt{1+|v|^{2}}}
$$

The collision integral has the form

$$
\begin{aligned}
& Q(F . F)(v)=\frac{1}{2 v_{0}} \iiint \delta\left(U^{2}-1\right) \delta\left(U^{\prime 2}-1\right) \delta\left(V^{\prime 2}-1\right) \\
& \cdot \delta^{(4)}\left(U+V-U^{\prime}-V^{\prime}\right) s \sigma(s, \theta)\left[F\left(u^{\prime}\right) F\left(v^{\prime}\right)-F(u) F(v)\right] d^{4} U d^{4} U^{\prime} d^{4} V
\end{aligned}
$$

where $U^{2}=U \cdot U=u_{0}^{2}-u^{2}, u^{2}=u_{1}^{2}+u_{2}^{2}+u_{3}^{2}, \delta$ is the delta function in one variable, $\delta^{(4)}$ is the delta function in four variables, and all the $F$ are evaluated at the same space-time point $(t, x)$. Furthermore $\sigma(s, \theta)$ is called the differential cross section or the scattering kernel; it is a function of variables $s$ and $\theta$ which will be defined later. The delta functions express the conservation of momentum and energy:

$$
\begin{aligned}
u^{\prime}+v^{\prime} & =u+v, \\
\sqrt{1+\left|u^{\prime}\right|^{2}}+\sqrt{1+\left|v^{\prime}\right|^{2}} & =\sqrt{1+|u|^{2}}+\sqrt{1+|v|^{2}} .
\end{aligned}
$$

Of course, the 12 -fold integral (0.2) defining $Q$ may be reduced to a 5 -fold integral by carrying out the delta function integrations (see Section 1).

A relativistic Maxwellian is characterized as a particle distribution $\mu(v)$ which minimizes the entropy subject to constant mass, momentum and energy. It is an equilibrium solution of $(R B)_{0}$ since $Q(\mu, \mu)=0$, and it has the form

$$
\mu(v)=\exp \left(a+b \cdot v-c \sqrt{1+v^{2}}\right)
$$

where $a, b$ and $c>|b|$ are five parameters (constants). We consider a solution $F(t, x, v)$ of $(R B)_{0}$ which has period $2 \pi$ in each $x$ variable and satisfies an initial condition $F(0, x, v)=F_{0}(x, v)$. We shall assume that the initial distribution $F_{0}(x, v)$ is close to a Maxwellian $\mu(v)$. The 5 parameters $a, b, c$ are chosen so that $F^{0}$ and $\mu$ have the same total mass, energy and momentum:

$$
0=\iint\left(F^{0}-\mu\right) d x d v=\iint v\left(F^{0}-\mu\right) d x d v=\iint \sqrt{1+v^{2}}\left(F^{0}-\mu\right) d x d v
$$

where the integration is over $x \in \Omega=(0,2 \pi)^{3}$ and $v \in \mathbb{R}^{3}$. (See Appendix $I$ ). The following is a special case of our main result.

Theorem D. Let $\sigma(s, \theta)$ satisfy (1.16)-(1.18) below. Let $F^{0}(x, v)$ be a nonnegative continuous function which has period $2 \pi$ in each $x$-variable. Assume that $\mu(v)$ is a Maxwellian (0.5) and $c_{0}$ is a positive constant such that 


$$
\sup _{x}\left|F^{0}(x, v)-\mu(v)\right| \leq c_{0}\left(1+|v|^{2}\right)^{-\alpha / 2} \sqrt{\mu(v)}
$$

where $\alpha>\frac{1}{2}(3+\beta)$ is fixed. If $c_{0}$ is sufficiently small, then there exist constants $c_{1}$ and $h>0$ and a unique global continuous solution $F(t, x, v)$ of $(R B)_{0}$ for $0 \leq t<$ $\infty$ such that $F(0, x, v)=F^{0}(x, v)$ and

$$
\sup _{x}|F(t, x, v)-\mu(v)| \leq c_{1} e^{-h t}\left(1+v^{2}\right)^{-\alpha / 2} \sqrt{\mu(v)}
$$

for all $v \in \mathbb{R}^{3}$ and $t \in[0, \infty)$.

Furthermore we prove that if the $x$-derivatives of $F^{0}$ satisfy similar estimates, so do the $x$-derivatives of the solution $F$, and hence $F$ is smooth in $x$. We also prove analogous results using $L^{2}$ norms in $x$. As is well-known, the periodicity condition in $x$ implies that we have solved $(R B)_{0}$ in a box with specular boundary conditions.

The classical Boltzmann equation was first solved in the spatially homogeneous case by Carleman [4], and more generally by Grad [15] locally in time, and by Ukai [22] [23] and Nishida and Imai [19] globally in time near a Maxwellian. Important contributions to the global problem have also been made by Caflisch [3], Shizuta [20], Illner and Shinbrot [17] and many others. More recently, global weak solutions with arbitrary initial data have been constructed by DiPerna and Lions [8]. All of the proofs of stability are based on the fact that the linearized equation possesses some dissipation, due to the decrease of the entropy.

In the relativistic case, we write the linearized equation of $(R B)_{0}$ as

$$
\partial_{t} f+\hat{v} \cdot \nabla_{x} f+\nu(v) f+K f=0
$$

where $K$ is a certain integral operator in $v$, and $\nu(v)>0$ is a scalar function of $v$ which represents the dissipation. In order to prove dissipation on the operator level in the sense of spectral theory, some compactness property of the solutions of $(0.8)$ is required. Some compactness in the $v$ variable follows from the form of $K$, while some compactness in the $x$ variable follows from the fact that $v$-averages of solutions of transport equations tend to be $x$-smoothing. The precise condition we use is a kind of relative compactness of operators, called $A$-smoothing. To a certain extent we follow the abstract approach that Shizuta [20] applied to the classical Boltzmann equation.

For background on the relativistic equation we mention the books of Stewart [21] and deGroot et. al [7]. There are some analyses of $(R B)_{0}$ by Cercignani and Majorana [6]. The linearized relativistic equation $(0.8)$ is solved by 
Dudynski and Ekiel-Jezewska [9].

In $§ 1$ we explicitly write the equation and derive the collision invariants and entropy inequality. Then we linearize the equation, and we formulate the function spaces $Y$ and the main theorem. In $\S 2$ we essentially show that the (nonlinear) collision integral is bounded on $Y$. The relativistic energy plays an important role here.

In $\S 3$ we estimate the kernel of the linearized collision operator. The relativistic estimates, which are related to those of Dudynski et. al. [9], are quite distinct from the classical ones of Grad and others. The smoothing property of the linearized equation is proved in $\$ 4$, following in part the approach of Shizuta [20]. Finally in $\S 5$ we solve the nonlinear problem by iteration by making use of the exponential decay of the linearized problem. In particular, we get a classical solution of $(R B)_{0}$ where each term is at least a continuous function.

In a succeeding paper we shall use the methods developed here to solve a relativistic Vlasov-Boltzmann system near equilibrium.

We thank R. Caflisch and A. M. Anile for providing us with several references on the mathematical theory of the Boltzmann equation.

\section{$\S 1$. Formulation}

We begin by defining the remaining variables in the collision integral $(0.2)$. We define

$$
\begin{aligned}
s & =(U+V)^{2}=\left(u_{0}+v_{0}\right)^{2}-|u+v|^{2} \\
& =2 u_{0} v_{0}-2 u \cdot v+u_{0}^{2}-|u|^{2}+v_{0}^{2}-|v|^{2} \\
& =2\left(\sqrt{1+|u|^{2}} \sqrt{1+|v|^{2}}-u \cdot v+1\right) \\
4 g^{2} & =-(U-V)^{2}=-\left(u_{0}-v_{0}\right)^{2}+|u-v|^{2} \\
& =2 u_{0} v_{0}-2 u \cdot v-u_{0}^{2}+|u|^{2}-v_{0}^{2}+|v|^{2} \\
& =2\left(\sqrt{1+|u|^{2}} \sqrt{1+|v|^{2}}-u \cdot v-1\right) \\
& =s-4,
\end{aligned}
$$

and

$$
\cos \theta=\frac{(V-U) \cdot\left(V^{\prime}-U^{\prime}\right)}{(V-U)^{2}} .
$$

Furthermore, we define the Møller velocity as the scalar $v_{M}$ given by

$$
v_{M}^{2}=|\hat{v}-\hat{u}|^{2}-|\hat{v} \wedge \hat{u}|^{2}=\frac{s(s-4)}{4 v_{0}^{2} u_{0}^{2}}
$$


or

$$
v_{M}=\frac{2 g \sqrt{1+g^{2}}}{v_{0} u_{0}} .
$$

The two expressions for $v_{M}^{2}$ are equal because

$$
\begin{aligned}
\frac{1}{4} s(s-4) & =s g^{2}=\left(u_{0} v_{0}-u \cdot v+1\right)\left(u_{0} v_{0}-u \cdot v-1\right) \\
& =|u|^{2}+|v|^{2}+|u|^{2}|v|^{2}-2 u_{0} v_{0} u \cdot v+(u \cdot v)^{2} \\
& =u_{0}^{2}|v|^{2}+v_{0}^{2}|u|^{2}-2 u_{0} v_{0} u \cdot v-|u \wedge v|^{2} \\
& =u_{0}^{2} v_{0}^{2}\left[\frac{|v|^{2}}{v_{0}^{2}}+\frac{|u|^{2}}{u_{0}^{2}}-2 \frac{u}{u_{0}} \cdot \frac{v}{v_{0}}-\left|\frac{u}{u_{0}} \wedge \frac{v}{v_{0}}\right|^{2}\right] .
\end{aligned}
$$

In deGroot et al. [7] the delta functions in the collision integral are carried out, resulting in the equation

$$
\partial_{t} F+\hat{v} \cdot \nabla_{x} F=\int_{R^{3}} \int_{s^{2}} v_{M} \sigma(g, \theta) \cdot\left[F\left(u^{\prime}\right) F\left(v^{\prime}\right)-F(u) F(v)\right] d \Omega d u
$$

where $d \Omega$ is the element of surface area on $S^{2}$ and we have written $\sigma$ as a function of $g$ and $\theta$. The variables $u, v, u^{\prime}, v^{\prime}$ are related by the equations (0.3) and (0.4). These equations allow $u^{\prime}$ and $v^{\prime}$ to be written in terms of $u$ and $v$ and a pair of variables $\theta$ and $\varphi$ which run over the unit sphere $S^{2}$. Equation $(R B)$ is the result of one such representation. A slightly different representation is given in Appendix II.

We remark that in the center-of-mass coordinate frame where $u+v=0$, $\sqrt{s}$ is the energy, $-2 g$ is the relative momentum, and $\theta$ is the scattering angle.

In the classical limit, where $|u|+|v| \ll 1$, we have $s \sim 4+|u-v|^{2}$, so that $v_{M} \sim|u-v|$ and $(R B)$ is the classical Boltzmann equation.

\section{Invariants}

Next we discuss the collision invariants and the entropy. While the main discussion is standard, there are some differences from the classical theory. Define the symmetrized collision operator

$$
Q^{*}(f, g)=\frac{1}{2} \iint v_{M} \sigma\left[f\left(v^{\prime}\right) g\left(u^{\prime}\right)+f\left(u^{\prime}\right) g\left(v^{\prime}\right)-f(v) g(u)-f(u) g(v)\right] d u d \Omega .
$$

The collision operator in $(R B)$ is $Q(F, F)=Q^{*}(F, F)$.

Lemma 1.1. For any functions $\varphi(v), f(v)$ and $g(v)$, sufficiently smooth and small at infinity, we have the four identities 


$$
\begin{gathered}
2 \int Q^{*}(f, g) \varphi d v=\iiint v_{M} \sigma\left[f\left(v^{\prime}\right) g\left(u^{\prime}\right)+f\left(u^{\prime}\right) g\left(v^{\prime}\right)-f(v) g(u)\right. \\
-f(u) g(v)]\left[\begin{array}{c}
\varphi(v) \\
\varphi(u) \\
-\varphi\left(v^{\prime}\right) \\
-\varphi\left(u^{\prime}\right)
\end{array}\right] d u d v d \Omega
\end{gathered}
$$

The proof is given in Appendix II.

Lemma 1.2. For $f, g$ as in Lemma 1.1, the collision operator satisfies

(a) $\int Q^{*}(f, g) d v=0, \int v Q^{*}(f, g) d v=0, \int \sqrt{1+v^{2}} Q^{*}(f, g) d v=0$.

(b)

$$
\int Q(f, f)(1+\log f) d v \geq 0 \text {. }
$$

Proof. For (a) we choose successively $\varphi(v)=1, \varphi(v)=v_{j}(j=1,2,3)$, and $\varphi(v)=\sqrt{1+v^{2}}$. The identities follow easily. For (b) we choose $\varphi(v)=1+\log f$ and add the four identities in (1.6) to get

$$
\begin{aligned}
& \int Q(f, f)(1+\log f) d v=\iiint v_{M} \sigma\left[f\left(u^{\prime}\right) f\left(v^{\prime}\right)-f(u) f(v)\right] \\
& \quad \cdot\left[\log f(u)+\log f(v)-\log f\left(u^{\prime}\right)-\log f\left(v^{\prime}\right)\right] d u d v d \Omega \\
& =\iiint v_{M} \sigma\left[f\left(u^{\prime}\right) f\left(v^{\prime}\right)-f(u) f(v)\right]\left[\log \{f(u) f(v)\}-\log \left\{f\left(u^{\prime}\right) f\left(v^{\prime}\right)\right\}\right] d u d v d \Omega .
\end{aligned}
$$

This is non-positive because the logarithm is increasing.

It follows from Lemma 1.2 that, for the solutions of $(R B)$, the mass $\int F d v d x$, the momentum $\int v F d v d x$, and the energy $\int \sqrt{1+v^{2}} F d v d x$ are invariants. Furthermore $\int F \log F d v d x$ is a non-increasing function of $t$.

\section{Linearization}

We shall now state our stability results in greater generality. First of all, we may normalize the Maxwellian to be

$$
\mu(v)=\exp \left(-\sqrt{1+v^{2}}\right)
$$

See Appendix I. It is convenient to change variables from $F$ to $f$ by

$$
F=\mu+\sqrt{\mu} f .
$$

Substituting (1.8) into $(R B)$, we have

$$
\partial_{t} f+\hat{v} \cdot \nabla_{x} f=\frac{1}{\sqrt{\mu}} Q(\mu+\sqrt{\mu} f, \mu+\sqrt{\mu} f)
$$




$$
=\frac{1}{\sqrt{\mu}}[Q(\mu, \sqrt{\mu} f)+Q(\sqrt{\mu} f, \mu)]+\frac{1}{\sqrt{\mu}} Q(\sqrt{\mu} f, \sqrt{\mu} f)
$$

or

$$
\partial_{t} f+\hat{v} \cdot \nabla_{x} f=-\nu(v) f-K f+\tilde{Q}(f, f)
$$

where

$$
\begin{aligned}
\tilde{Q}(f, f) & =\frac{1}{\sqrt{\mu}} Q(\sqrt{\mu} f, \sqrt{\mu} f) \\
& =\iint \frac{g \sqrt{1+g^{2}}}{u_{0} v_{0}} \sigma(g, \theta) \sqrt{\mu(u)}\left[f\left(u^{\prime}\right) f\left(v^{\prime}\right)-f(u) f(v)\right] d u d \Omega, \\
\nu(v) & =\iint \frac{g \sqrt{1+g^{2}}}{u_{0} v_{0}} \sigma(g, \theta) \mu(u) d u d \Omega
\end{aligned}
$$

and

$$
\begin{aligned}
K f(v)= & \iint \frac{g \sqrt{1+g^{2}}}{u_{0} v_{0}} \sigma(g, \theta) \sqrt{\mu(u)}\left[-\sqrt{\mu\left(u^{\prime}\right)} f\left(v^{\prime}\right)-\sqrt{\mu\left(v^{\prime}\right)} f\left(u^{\prime}\right)\right. \\
& +\sqrt{\mu(v)} f(u)] d u d \Omega .
\end{aligned}
$$

If $F$ is a solution of $(R B)$ and (0.5a) and (1.8) hold, then we have

$$
0=\iint \sqrt{\mu} f d v d x=\iint v \sqrt{\mu} f d v d x=\iint \sqrt{1+|v|^{2}} \sqrt{\mu} f d v d x .
$$

The entropy implies the following dissipative property of the linearized operator $-\nu-K$.

\section{Lemma 1.3.}

$$
\int(-\nu-K) f(v) \cdot f(v) d v<0
$$

for any function $f(v)$ which satisfies (1.11a).

Proof. We notice that

$$
(-\nu-K) f=\frac{1}{\sqrt{\mu}}[Q(\mu, \sqrt{\mu} f)+Q(\sqrt{\mu} f, \mu)]=\frac{1}{\sqrt{\mu}} Q^{*}(\mu, \sqrt{\mu} f) .
$$

In (1.6) we substitute $\varphi=\mu^{-1 / 2} f$ and add the four identities to get

$$
\begin{aligned}
4 \int & (-\nu-K) f \cdot f d v \\
= & \iiint v_{M} \sigma\left[\sqrt{\mu\left(v^{\prime}\right)} \mu\left(u^{\prime}\right) f\left(v^{\prime}\right)+\sqrt{\mu\left(u^{\prime}\right)} \mu\left(v^{\prime}\right) f\left(u^{\prime}\right)-\sqrt{\mu(v)} \mu(u) f(v)\right. \\
& -\sqrt{\mu(u)} \mu(v) f(u)] \cdot\left[\frac{f(v)}{\sqrt{\mu(v)}}+\frac{f(u)}{\sqrt{\mu(u)}}-\frac{f\left(v^{\prime}\right)}{\sqrt{\mu\left(v^{\prime}\right)}}-\frac{f\left(u^{\prime}\right)}{\sqrt{\mu\left(u^{\prime}\right)}}\right] d u d v d \Omega
\end{aligned}
$$




$$
\begin{aligned}
& =-\iiint v_{M} \sigma \mu(u) \mu(v)\left[\frac{f\left(v^{\prime}\right)}{\sqrt{\mu\left(v^{\prime}\right)}}+\frac{f\left(u^{\prime}\right)}{\sqrt{\mu\left(u^{\prime}\right)}}-\frac{f(u)}{\sqrt{\mu(u)}}-\frac{f(v)}{\sqrt{\mu(v)}}\right]^{2} d u d v d \Omega \\
& \leq 0 \quad \text { since } \quad \mu\left(u^{\prime}\right) \mu\left(v^{\prime}\right)=\mu(u) \mu(v) .
\end{aligned}
$$

This integral vanishes if and only if $f(v)$ has the form

$$
f(v)=\left\{a+b \cdot v+c \sqrt{1+v^{2}}\right\} \sqrt{\mu(v)} .
$$

Because of (1.11a), this integral is strictly negative.

\section{Spaces}

Now we define the spaces to which $f$ will belong. In the $x$ variable we use the spaces $C^{k}$ of periodic functions whose $k$-th derivatives are continuous, or the Sobolev spaces $H^{k}$ of periodic functions whose $k$-th derivatives belong to $L^{2}$. Let $X$ denote either $C^{k}$ for $k \geq 0$ or $H^{k}$ for $k \geq 2$. Let $\rho_{\alpha}(v)=\left(1+|v|^{2}\right)^{\alpha / 2}$. Define $G_{\alpha}(X)$ as the space of continuous functions $f: \mathbb{R}^{3} \rightarrow X$ for which

$$
\|f\|=\sup _{v \in R^{3}} \rho_{\sigma}(v)|f(v)|_{X}<\infty .
$$

Furthermore define $G_{\alpha}^{0}(X)$ as the closed subspace of $G_{\alpha}(X)$ for which

$$
\lim _{|v| \rightarrow \infty} \rho_{\infty}(v)|f(v)|_{X}=0,
$$

furnished with the same norm. Let

$$
Y=\text { either } G_{a}^{0}\left(H^{l}\right) \text { or } G_{a}^{0}\left(C^{k}\right)
$$

where $\alpha \geq 0, k \geq 0$ and $l \geq 2$.

\section{Hypothesis on $\sigma$.}

We now state the hypothesis on the collision cross-section $\sigma(g, \theta)$. It should satisfy

$$
c_{1} \frac{g^{\beta+1}}{1+g} \sin ^{\gamma} \theta \leq \sigma(g, \theta) \leq c_{2}\left(g^{\beta}+g^{-\delta}\right) \sin ^{\gamma} \theta
$$

and

$$
\left|\frac{\partial \sigma}{\partial g}\right| \leq c_{3}\left(g^{\beta^{\prime}}+g^{-\delta^{\prime}}\right) \sin { }^{\gamma^{\prime} \theta}
$$

where $c_{1}, c_{2}$ and $c_{3}$ are positive constants, $0 \leq \delta<\frac{1}{2}, 0 \leq \beta<2-2 \delta, 0 \leq \delta^{\prime}<4$, $\beta^{\prime} \geq 0, \gamma^{\prime}>-2$, and either $r \geq 0$ or

$$
|r|<\min \left\{2-\beta, \frac{1}{2}-\delta, \frac{1}{3}(2-2 \delta-\beta)\right\} \text {. }
$$


Now we can state the main theorem, which is more general than Theorem 0.

Theorem 1.1. Assume that $\sigma(g, \theta)$ satisfies (1.16) and (1.17). Let $Y$ be either of the spaces (1.15), where $\alpha>\frac{1}{2}(3+\beta)$. If $f^{0} \in Y$ satisfies (1.11a) and $\left\|f^{0}\right\|_{Y}$ is sufficiently small, then there exists $h>0$ and a unique global solution $f(t, x, v)$ of $(R B)$ which is periodic in $x$ and satisfies $f(0, x, v)=f^{0}(x, v), f \in$ $C([0, \infty) ; Y)$ and

$$
\|f(t)\|_{Y} \leq c_{4}\left\|f^{0}\right\|_{Y} e^{-h t}, \quad 0 \leq t<\infty .
$$

\section{$\S 2$. Estimates on the Collision Operator}

We have seen in (1.9) that the nonlinear collision operator has the form

$$
\begin{aligned}
\tilde{Q}(f, f) & =\iint_{|\Omega|=1} v_{M} \sigma(g, \theta) e^{-u_{0} / 2}\left(f\left(u^{\prime}\right) f\left(v^{\prime}\right)-f(u) f(v)\right) d u d \Omega \\
& \equiv \tilde{Q}_{\text {gain }}-\tilde{Q}_{\text {loss }}
\end{aligned}
$$

where the "gain" term contains the primes; the loss term does not. The major result of this section is the following.

Theorem 2.1. Assume $\sigma$ satisfies (1.16)-(1.18) and consider any of the spaces

$$
G_{a}^{0}\left(H^{l}\right) \text { or } G_{a}^{0}\left(C^{k}\right)
$$

for $\alpha \geq \beta / 2, l \geq 2, k \geq 0$. Let $\|\cdot\|_{\infty}$ be the norm in any one of these spaces. If $f \in G_{\alpha}^{0}$, then $\tilde{Q}(f, f) \in G_{\alpha-\beta / 2}^{0}$ and

$$
\|\tilde{Q}(f, f)\|_{\alpha-\beta / 2} \leq c\|f\|_{\alpha}^{2} .
$$

The map $\tilde{Q}$ is continuous from $G_{\alpha}^{0}$ into $G_{\alpha-\beta / 2}^{0}$; in fact,

$$
\|\tilde{Q}(f, f)-\tilde{Q}(g, g)\|_{\alpha-\beta / 2} \leq c\left(\|f\|_{\alpha}+\|g\|_{\alpha}\right)\|f-g\|_{\alpha} .
$$

Here are two lemmas to be frequently used below.

Lemma 2.1. There is a constant $c$, depending only on the parameters in (1.16)-(1.18), such that

$$
\iint_{|\Omega|=1} e^{-(1 / 2) u_{0}} \sigma(g, \theta) d \Omega d u \leq c v_{0}^{\beta / 2}
$$

Proof. Using (1.16) we have 


$$
\begin{aligned}
\int_{|\Omega|=1} \sigma(g, \theta) d \Omega & \leq c \int_{0}^{2 \pi}\left(g^{-\delta}+g^{\beta}\right) \sin ^{1-\gamma} \theta d \theta \\
& \leq c\left(g^{-\delta}+g^{\beta}\right) .
\end{aligned}
$$

From its definition, $g^{2} \leq c u_{0} v_{0}$ and from Lemma 3.1, $g \geq \frac{c|u-v|}{u_{0}^{1 / 2} v_{0}^{1 / 2}}$. Thus

$$
\int_{|\Omega|=1} \sigma d \Omega \leq c\left[\frac{u_{0}^{\delta / 2} v_{0}^{\delta / 2}}{|u-v|^{\delta}}+u_{0}^{\beta / 2} v_{0}^{\beta / 2}\right]
$$

so that

$$
\iint_{|\Omega|=1} e^{-(1 / 2) u_{0}} \sigma d \Omega d u \leq c v_{0}^{\beta / 2}+c v_{0}^{\delta / 2} \int \frac{u_{0}^{\delta / 2} e^{-u_{0} / 2}}{|u-v|^{\delta}} d u
$$

The remaining integral certainly converges; the only question is its growth in $|v|$. On the sets $\{u:|u|>2|v|\}$ and $\left\{u:|u|<\frac{|v|}{2}\right\}$ we have $|u-v| \geq \frac{|v|}{2}$, and thus the contribution of these sets to the second expression is bounded. On the set $\left\{\frac{|v|}{2} \leq|u| \leq 2|v|\right\}$ we write

$$
v_{0}^{\delta / 2} \int_{|v| / 2 \leqq|u| \leqq 2|v|} \frac{u_{0}^{\delta / 2} e^{-u_{0} / 2}}{|u-v|^{\delta}} d u \leq c \int \frac{u_{0}^{\delta} e^{-u_{0} / 2}}{|u-v|^{\delta}} d u .
$$

This expression is bounded as we see from breaking it up at $|u-v|=1$.

\section{Lemma 2.2.}

$$
\rho_{\alpha}\left(u^{\prime}\right) \rho_{\alpha}\left(v^{\prime}\right) \geq c_{\alpha} \rho_{\alpha}(v) \text { for some } c_{\alpha}>0 \text {. }
$$

Also, for $|v|$ large, say $|v| \geq 4$, either $\left|u^{\prime}\right| \geq|v| / 4$ or $\left|v^{\prime}\right| \geq|v| / 4$. Here $c_{\text {w }}$ depends only on $\alpha$, and $u^{\prime}, v^{\prime}$ are defined in (0.3), (0.4).

Proof. Energy conservation implies

$$
\sqrt{1+|v|^{2}} \leq \sqrt{1+\left|u^{\prime}\right|^{2}}+\sqrt{1+\left|v^{\prime}\right|^{2}}
$$

and hence

$$
\begin{aligned}
1+|v|^{2} & \leq 2\left[1+\left|u^{\prime}\right|^{2}+1+\left|v^{\prime}\right|^{2}\right]=4+2\left(\left|u^{\prime}\right|^{2}+\left|v^{\prime}\right|^{2}\right) \\
& \leq 4\left(1+\left|u^{\prime}\right|^{2}+\left|v^{\prime}\right|^{2}\right) \leq 4\left(1+\left|u^{\prime}\right|^{2}\right)\left(1+\left|v^{\prime}\right|^{2}\right) .
\end{aligned}
$$

Taking the $\alpha / 2$ power, we get the conclusion, where $\rho_{\alpha}$ is defined in (1.13).

For the second statement, consider the invariant energy

$$
e \equiv \sqrt{1+|u|^{2}}+\sqrt{1+|v|^{2}}=\sqrt{1+\left|u^{\prime}\right|^{2}}+\sqrt{1+\left|v^{\prime}\right|^{2}}
$$

Thus $e>\sqrt{1+|v|^{2}}>|v|$. Let $|v| \geq 4$. If the assertion were false, then 


$$
\begin{aligned}
e & =\sqrt{1+\left|u^{\prime}\right|^{2}}+\sqrt{1+\left|v^{\prime}\right|^{2}}<2 \sqrt{1+\frac{|v|^{2}}{16}} \\
& \leq 2 \sqrt{2 \cdot \frac{|v|^{2}}{16}}=\frac{\sqrt{2}}{2}|v|<|v|
\end{aligned}
$$

a contradiction.

Proof of Theorem 2.1. We consider the gain and loss terms separately. From Lemma 3.1 below, we know that $v_{M}$ is bounded. Thus in the representative case $k=2$,

$$
\begin{aligned}
& \sum_{|\lambda| \leq 2}\left|D_{x}^{\lambda} \tilde{Q}_{\text {loss }}(f, f)\right| \leq c \iint_{|\Omega|=1} \sigma e^{-u_{0} / 2} \cdot\left[|f(u) f(v)|+\left|f_{x}(u)\right||f(v)|\right. \\
& \left.\quad+\left|f(u) f_{x}(v)\right|+\left|f_{x x}(u) f(v)\right|+\left|f_{x}(u) f_{x}(v)\right|+\left|f(u) f_{x x}(v)\right|\right] d \Omega d u \\
& \quad \equiv L_{1}+L_{2}+\cdots+L_{6} .
\end{aligned}
$$

The gain term satisfies the same bound with $u, v$ replaced by $u^{\prime}, v^{\prime}$ in each argument of $f$. Thus we write

$$
\sum_{|\lambda| \leq 2}\left|D_{x}^{\lambda} \tilde{Q}_{\text {gain }}\right| \leq c\left[G_{1}+G_{2}+\cdots+G_{6}\right]
$$

where e.g.

$$
\begin{aligned}
& G_{1}=\iint_{|\Omega|=1} \sigma e^{-u_{0} / 2}\left|f\left(u^{\prime}\right)\right|\left|f\left(v^{\prime}\right)\right| d u d \Omega, \\
& G_{2}=\iint_{|\Omega|=1} \sigma e^{-u_{0} / 2}\left|f_{x}\left(u^{\prime}\right)\right|\left|f\left(v^{\prime}\right)\right| d u d \Omega,
\end{aligned}
$$

etc.

We begin with $f \in Y=G_{a}^{0}\left(C^{k}\right)$. Consider the loss terms. For instance,

$$
\begin{aligned}
\left|L_{6}\right| & =c\left|f_{x x}(v)\right| \iint \sigma(g, \theta) e^{-u_{0} / 2}|f(u)| d \Omega d u \\
& \leq c \rho_{\alpha}^{-1}(v)\left[\rho_{\alpha}(v)|f(v)| c^{2}\right] \cdot \sup _{u}\left[\rho_{\alpha}(u)|f(u)|_{\left.c^{2}\right]}\right] \int \frac{\sigma e^{-u_{0} / 2}}{\rho_{\alpha}(u)} d \Omega d u .
\end{aligned}
$$

By Lemma 2.1, the last integral is $O\left(|v|^{\beta / 2}\right)$ so that

$$
\rho_{\alpha}(v)\left|L_{6}\right| \leq c|v|^{\beta / 2} \cdot\|f\|_{\alpha} \cdot \rho_{\alpha}(v)|f(v)|_{c^{2}} .
$$

Hence $\rho_{\alpha-(\beta / 2)}(v)\left|L_{6}\right| \leq c\|f\|_{\alpha} \cdot \rho_{\alpha}(v)|f(v)|_{c^{2}}$. Clearly, each of the loss terms satisfies the same bound, so that we get directly

$$
\rho_{\alpha-(\beta / 2)}(v)\left|\tilde{Q}_{\text {loss }}(f, f)(v)\right|_{c^{2}} \leq c\|f\|_{\alpha} \cdot \rho_{\alpha}(v)|f(v)|_{c^{2}}
$$

for $f \in Y=G_{\alpha}^{0}\left(C^{2}\right)$. It follows that $\tilde{Q}_{\text {loss }}(f, f) \in G_{\alpha-(\beta / 2)}^{0}\left(C^{2}\right)$ and 


$$
\left\|\tilde{Q}_{\text {loss }}(f, f)\right\|_{\alpha-(\beta / 2)} \leq c\|f\|_{\alpha} .
$$

Now consider the gain terms, still with $Y$ as above. A representative term is $G_{6}$, for which we have the estimate

$$
\begin{aligned}
\left|G_{6}\right| & \leq \iint \sigma e^{-u_{0} / 2}\left|f\left(u^{\prime}\right) f_{x x}\left(v^{\prime}\right)\right| d u d \Omega \\
& \leq \rho_{\alpha}^{-1}(v) \iint \sigma e^{-u_{0} / 2}\left[\rho_{\alpha}\left(u^{\prime}\right)\left|f\left(u^{\prime}\right)\right|\right]\left[\rho_{\alpha}\left(v^{\prime}\right)\left|f_{x x}\left(v^{\prime}\right)\right|\right] d u d \Omega
\end{aligned}
$$

where we have used Lemma 2.2. By Lemma 2.1, we get

$$
\rho_{\alpha}(v)\left|G_{6}\right| \leq c \rho_{\beta / 2}(v) \cdot\left[\sup _{u, \Omega} \rho_{\alpha}\left(u^{\prime}\right)\left|f\left(u^{\prime}\right)\right| c^{2}\right] \cdot\left[\sup _{u, \Omega} \rho_{\alpha(}\left(v^{\prime}\right)\left|f\left(v^{\prime}\right)\right|_{\left.c^{2}\right]}\right] .
$$

Each of the gain terms is so estimable. Thus we get

$$
\rho_{\alpha-(\beta / 2)}(v)\left|\tilde{Q}_{\text {gain }}(f, f)\right|_{c^{2}} \leq c\left[\operatorname { s u p } _ { u , | \Omega | = 1 } \rho _ { \alpha } ( u ^ { \prime } ) | f ( u ^ { \prime } ) | _ { c ^ { 2 } ] } \left[\sup _{u,|\Omega|=1} \rho_{\alpha}\left(v^{\prime}\right)\left|f\left(v^{\prime}\right)\right|_{\left.c^{2}\right]}\right.\right.
$$

It follows immediately that

$$
\left\|\tilde{Q}_{\text {gain }}(f, f)\right\|_{\alpha-(\beta / 2)} \leq c\|f\|_{\alpha}^{2} .
$$

It remains to show that

$$
\rho_{\alpha-\beta / 2}(v)\left|\tilde{Q}_{\text {gain }}(f, f)(v)\right|_{c^{2}} \rightarrow 0 \text { as }|v| \rightarrow \infty .
$$

Returning to (2.12), we assume $|v|$ is large and partition the integral for $G_{6}$.

$$
c\left[\int_{\left\{\left|u^{\prime}\right| \geq|v| / 4\right\}}^{\rho_{\alpha}(v)\left|G_{6}\right| \leq}+\int_{\left\{\left|v^{\prime}\right| \geq|v| / 4\right\}}\right] \sigma e^{-u_{0} / 2}\left[\rho_{\alpha}\left(u^{\prime}\right)\left|f\left(u^{\prime}\right)\right|_{\left.c^{2}\right]}\right]\left[\rho_{\alpha}\left(v^{\prime}\right)\left|f\left(v^{\prime}\right)\right| c^{2}\right] d \Omega d u .
$$

In the first integral, the expression $\rho_{\alpha}\left(u^{\prime}\right)\left|f\left(u^{\prime}\right)\right|_{c^{2 \rightarrow 0}}$ uniformly in $(u, \Omega)$ as $|v| \rightarrow \infty$ by Lemma 2.2. The second factor

$$
\rho_{\alpha}\left(v^{\prime}\right)\left|f\left(v^{\prime}\right)\right|_{c^{2}}
$$

is bounded above by $\|f\|_{Y}$, and the remaining integral is $O\left(|v|^{\beta / 2}\right)$ by Lemma 2.1. Thus we have

$$
\rho_{\alpha-(\beta / 2)}(v)\left|G_{6}\right|=o(1) \cdot\|f\|_{Y} \rightarrow 0 \quad \text { as } \quad|v| \rightarrow \infty .
$$

As noted above, each of the gain terms is estimable exactly as is $G_{6}$ in (2.17) and (2.18). This observation together with the estimate (2.11) for $\tilde{Q}_{\text {loss }}(f, f)$ prove the theorem in the case $Y=G_{\alpha}^{0}\left(C^{2}\right), \alpha \geq \beta / 2$. 
Now we turn to the more complicated case $f \in Y=G_{a}^{0}\left(H^{2}\right), \alpha \geq \beta / 2$. We begin with the typical term $G_{4}$ :

$$
\begin{aligned}
\left\|G_{4}\right\|_{L^{2}}^{2} \leq & \int\left|\int e^{-u_{0} / 2} \sigma f_{x x}\left(t, x, u^{\prime}\right) f\left(t, x, v^{\prime}\right) d u d \Omega\right|^{2} d x \\
\leq & \rho_{\alpha}^{-2}(v) \int\left|\int e^{-u_{0} / 2} \sigma \rho_{\alpha}\left(u^{\prime}\right) f_{x x}\left(t, x, u^{\prime}\right) \rho_{\alpha}\left(v^{\prime}\right) f\left(t, x, v^{\prime}\right) d u d \Omega\right|^{2} d x \\
\leq & \rho_{\alpha}^{-2}(v) \int\left(\int e^{-u_{0} / 2} \sigma \rho_{\alpha}^{2}\left(u^{\prime}\right) f_{x x}^{2}\left(t, x, u^{\prime}\right) d u d \Omega\right) \\
& \cdot\left(\int e^{-u_{0} / 2} \sigma \rho_{\alpha}^{2}\left(v^{\prime}\right) f^{2}\left(t, x, v^{\prime}\right) d u d \Omega\right) d x \\
\leq & \rho_{\alpha}^{-2}(v)\left[\int e^{-u_{0} / 2} \sigma \rho_{\alpha}^{2}\left(v^{\prime}\right) \sup _{x} f^{2}\left(t, x, v^{\prime}\right) d u d \Omega\right] \\
& \cdot \iint e^{-u_{0} / 2} \sigma \rho_{\alpha}^{2}\left(u^{\prime}\right) f_{x x}^{2}\left(t, x, u^{\prime}\right) d x d u d \Omega \\
\leq & c \rho_{\infty}^{-2}(v) \int e^{-u_{0} / 2} \sigma \rho_{\alpha}^{2}\left(v^{\prime}\right)\left\|f\left(\cdot, v^{\prime}\right)\right\|_{H^{2}}^{2} d u d \Omega \\
& \cdot \int e^{-u_{0} / 2} \sigma \rho^{2}\left(u^{\prime}\right)\left\|f\left(\cdot, u^{\prime}\right)\right\|_{H^{2}}^{2} d u d \Omega \\
\leq & c \rho_{\alpha}^{-2}(v)\left(\int e^{-u_{0} / 2} \sigma d u d \Omega\right)\left(\int e^{-u_{0} / 2} \sigma \rho_{\alpha}^{4}\left(v^{\prime}\right)\left\|f\left(\cdot, v^{\prime}\right)\right\|_{H^{2}}^{4} d u d \Omega\right)^{1 / 2} \\
& \cdot\left(\int e^{-u_{0} / 2} \sigma \rho_{\alpha}^{4}\left(u^{\prime}\right)\left\|f\left(\cdot, u^{\prime}\right)\right\|_{H^{2}}^{4} d u d \Omega\right)^{1 / 2} \cdot
\end{aligned}
$$

Thus we have by Lemma 2.1,

$$
\begin{aligned}
\rho_{\alpha}(v)\left\|G_{4}\right\|_{L^{2}} \leq \\
c|v|^{\beta / 4}\left(\int e^{-u_{0} / 2} \sigma \rho_{\omega}^{4}\left(v^{\prime}\right)\left\|f\left(\cdot, v^{\prime}\right)\right\|_{H^{2}}^{4} d u d \Omega\right)^{1 / 4} \\
\cdot\left(\int e^{-u_{0} / 2} \sigma \rho_{\omega}^{4}\left(u^{\prime}\right)\left\|f\left(\cdot, u^{\prime}\right)\right\|_{H^{2}}^{4} d u d \Omega\right)^{1 / 4} .
\end{aligned}
$$

Similarly the term $G_{6}$ admits the same estimate, since its integrand involves $f\left(u^{\prime}\right) f_{x x}\left(v^{\prime}\right)$; thus the sup over $x$ is taken on the $f\left(u^{\prime}\right)$-term. Moreover, terms $G_{1}, G_{2}$ and $G_{3}$ also satisfy the same estimate as that for $G_{4}$, since they involve only one derivative.

Hence we need only bound $G_{5}$, for which we have

$$
\begin{aligned}
\left\|G_{5}\right\|_{L^{2}}^{2} & \leq \int\left|\int e^{-u_{0} / 2} \sigma f_{x}\left(u^{\prime}\right) f_{x}\left(v^{\prime}\right) d u d \Omega\right|^{2} d x \\
& =\int\left|\int e^{-u_{0} / 2} \rho_{\alpha}\left(u^{\prime}\right) f_{x}\left(u^{\prime}\right) \rho_{\alpha}\left(v^{\prime}\right) f_{x}\left(v^{\prime}\right) \frac{d u d \Omega}{\rho_{\alpha}\left(u^{\prime}\right) \rho_{\alpha}\left(v^{\prime}\right)}\right|^{2} d x \\
& \leq \rho_{\alpha}^{-2}(v) \int \mid \int\left(\sigma^{1 / 2} e^{-u_{0} / 4}\right)\left(\sigma^{1 / 4} e^{-u_{0} / 8} \rho_{\alpha}\left(u^{\prime}\right) f_{x}\left(u^{\prime}\right)\right) \\
& \left.\cdot\left(\sigma^{1 / 4} e^{-u_{0} / 8} \rho_{\alpha}\left(v^{\prime}\right) f_{x}\left(v^{\prime}\right)\right) d u d \Omega\right|^{2} d x
\end{aligned}
$$




$$
\begin{aligned}
& \leq \rho_{\alpha}^{-2}(v) \int\left(\int e^{-u_{0} / 2} \sigma d u d \Omega\right)\left(\int \sigma e^{-u_{0} / 2} \rho_{a}^{4}\left(u^{\prime}\right) f_{x}^{4}\left(u^{\prime}\right) d u d \Omega\right)^{1 / 2} \\
& \cdot\left(\int \sigma e^{-u_{0} / 2} \rho_{\alpha}^{4}\left(v^{\prime}\right) f_{x}^{4}\left(v^{\prime}\right) d u d \Omega\right)^{1 / 2} d x \\
& \leq c|v|^{\beta / 2} \rho_{a}^{-2}(v)\left[\iint \sigma e^{-u_{0} / 2} \rho_{\alpha}^{4}\left(u^{\prime}\right) f_{x}^{4}\left(u^{\prime}\right) d x d u d \Omega\right]^{1 / 2} \\
& \cdot\left[\iint \sigma e^{-u_{0} / 2} \rho_{a}^{4}\left(v^{\prime}\right) f_{x}^{4}\left(v^{\prime}\right) d x d u d \Omega\right]^{1 / 2} \\
& \leq c|v|^{\beta / 2} \rho_{\alpha}^{-2}(v)\left[\int \sigma e^{-u_{0} / 2} \rho_{\alpha}^{4}\left(u^{\prime}\right)\left\|f\left(\cdot, u^{\prime}\right)\right\|_{H^{2}}^{4} d u d \Omega\right]^{1 / 2} \\
& \cdot\left[\int \sigma e^{-u_{0} / 2} \rho_{\alpha}^{4}\left(v^{\prime}\right)\left\|f\left(\cdot, v^{\prime}\right)\right\|_{H^{2}}^{4} d u d \Omega\right]^{1 / 2} .
\end{aligned}
$$

Therefore for $G_{5}$ we have the estimate

$$
\begin{aligned}
& \rho_{a \dot{ }}(v)\left\|G_{5}\right\|_{L^{2}} \leq c|v|^{\beta / 4}\left(\int \sigma e^{-u_{0} / 2} \rho_{a}^{4}\left(u^{\prime}\right)\left\|f\left(\cdot, u^{\prime}\right)\right\|_{H^{2}}^{4} d u d \Omega\right)^{1 / 4} \\
& \cdot\left(\int \sigma e^{-u_{0} / 2} \rho_{a}^{4}\left(v^{\prime}\right)\left\|f\left(\cdot, v^{\prime}\right)\right\|_{H^{2}}^{4} d u d \Omega\right)^{1 / 4}
\end{aligned}
$$

which is the same as the estimate (2.19) already obtained for $G_{4}$. Since all of the gain terms are so estimable, we have now shown that

$$
\begin{aligned}
\rho_{\alpha \prime-(\beta / 4)}(v) & \sum_{|\lambda| \leq 2}\left\|D_{x}^{\lambda} \tilde{Q}_{\text {gain }}(f, f)(v)\right\|_{L^{2}} \\
\leq c & \left(\int \sigma e^{-u_{0} / 2} \rho_{\alpha}^{4}\left(u^{\prime}\right)\left\|f\left(\cdot, u^{\prime}\right)\right\|_{H^{2}}^{4} d u d \Omega\right)^{1 / 4} \\
& \quad\left(\int \sigma e^{-u_{0} / 2} \rho_{\alpha}^{4}\left(v^{\prime}\right)\left\|f\left(\cdot, v^{\prime}\right)\right\|_{H^{2}}^{4} d u d \Omega\right)^{1 / 4} .
\end{aligned}
$$

The first of the integrals appearing here can be bounded as follows:

$$
\begin{aligned}
& \left(\int \sigma e^{-u_{0} / 2} \rho_{a}^{4}\left(u^{\prime}\right)\left\|f\left(\cdot, u^{\prime}\right)\right\|_{H^{2}}^{4} d u d \Omega\right)^{1 / 4} \\
& \quad \leq \sup _{u^{\prime}} \rho_{\alpha}\left(u^{\prime}\right)\left\|f\left(\cdot, u^{\prime}\right)\right\|_{H^{2}} \cdot\left(\int \sigma e^{-u_{0} / 2} d u d \Omega\right)^{1 / 4} \\
& \quad \leq c\|f\|_{Y} \cdot|v|^{\beta / 8} \leq c \rho_{\beta / 8}(v) \cdot\|f\|_{Y},
\end{aligned}
$$

as can the second. (2.21) then yields

$$
\rho_{\alpha-\beta / 2}(v) \sum_{|\lambda| \leq 2}\left\|D_{x}^{\lambda} \tilde{Q}_{\text {gain }}(f, f)(v)\right\|_{L^{2}} \leq c\|f\|_{Y}^{2}
$$

i.e.

$$
\left\|\tilde{Q}_{\text {gain }}(f, f)\right\|_{\alpha-\beta / 2} \leq c\|f\|_{\alpha}^{2}
$$

which is one of the two estimates desired.

It remains to show that, for $f \in G_{a}^{0}\left(H^{2}\right)$, 


$$
\rho_{\alpha-\beta / 2}(v)\left\|\tilde{Q}_{\text {gain }}(f, f)(v)\right\|_{H^{2}} \rightarrow 0 \text { as }|v| \rightarrow \infty .
$$

This is achieved by partitioning each of the integrals as was done previously, and by using Lemma 2.2. Explicitly, consider again the term $G_{4}$, and let $|v|$ be large. By Lemma 2.2, we can write

$$
\begin{aligned}
\left\|G_{4}\right\|_{L^{2}} \leq & {\left[\int\left|\int_{\left\{\left|u^{\prime}\right| \geq|v| / 4\right\}} e^{-u_{0} / 2} \sigma f_{x x}\left(u^{\prime}\right) f\left(v^{\prime}\right) d u d \Omega\right|^{2} d x\right]^{1 / 2} } \\
& +\left[\int\left|\int_{\left\{\left|v^{\prime}\right| \geq|v| / 4\right\}} e^{-u_{0} / 2} \sigma f_{x x}\left(u^{\prime}\right) f\left(v^{\prime}\right) d u d \Omega\right|^{2} d x\right]^{1 / 2} .
\end{aligned}
$$

Each of these integrals is bounded in exactly the same way which led to (2.18). In this manner, we obtain the following estimate, analogous to (2.20):

$$
\begin{aligned}
& \rho_{\alpha}(v)\left\|G_{4}\right\|_{L^{2}} \leq c|v|^{\beta / 4} \\
& \cdot\left[\left(\int_{\left\{\left|u^{\prime}\right| \geq|v| / 4\right\}} e^{-u_{0} / 2} \sigma \rho_{\alpha}^{4}\left(v^{\prime}\right)\left\|f\left(\cdot, v^{\prime}\right)\right\|_{H^{2}}^{4} d u d \Omega\right)^{1 / 4}\right. \\
& \cdot\left(\int_{\left\{\left|u^{\prime}\right| \geq|v| / 4\right\}} e^{-u_{0} / 2} \sigma \rho_{\alpha}^{4}\left(u^{\prime}\right)\left\|f\left(\cdot, u^{\prime}\right)\right\|_{H^{2}}^{4} d u d \Omega\right)^{1 / 4} \\
&+\left(\int_{\left\{\left|v^{\prime}\right| \geq|v| / 4\right\}} e^{-u_{0} / 2} \sigma \rho_{\alpha}^{4}\left(v^{\prime}\right)\left\|f\left(\cdot, v^{\prime}\right)\right\|_{H^{2}}^{4} d u d \Omega\right)^{1 / 4} \\
&\left.\cdot\left(\int_{\left\{\left|v^{\prime}\right| \geq|v| / 4\right\}} e^{-u_{0} / 2} \sigma \rho_{\alpha}^{4}\left(u^{\prime}\right)\left\|f\left(\cdot, u^{\prime}\right)\right\|_{H^{2}}^{4} d u d \Omega\right)^{1 / 4}\right] \\
& \leq c|v|^{\beta / 4}\left[|v|^{\beta / 8} \cdot\|f\|_{Y} \cdot|v|^{\beta / 8} \sup _{\left\{\left|u^{\prime}\right| \geq|v| / 4\right\}} \rho_{\alpha}\left(u^{\prime}\right)\left\|f\left(\cdot, u^{\prime}\right)\right\|_{H^{2}}\right. \\
&+\left.|v|^{\beta / 8} \cdot \sup _{\left\{\left|v^{\prime}\right| \geq|v| / 4\right\}} \rho_{\alpha}\left(v^{\prime}\right)\left\|f\left(\cdot, v^{\prime}\right)\right\|_{H^{2}} \cdot|v|^{\beta / 8}\|f\|_{Y}\right] \\
& \leq c|v|^{\beta / 2}\|f\|_{Y} \cdot o(1) .
\end{aligned}
$$

Therefore

$$
\rho_{\alpha-\beta / 2}(v)\left\|G_{4}\right\|_{L^{2}} \rightarrow 0 \text { as }|v| \rightarrow \infty .
$$

Now we recall that all other gain terms can be estimated as $G_{4}$ and $G_{5}$ can. We partition $G_{5}$ as we partitioned $G_{4}$ above, and proceed similarly with each integral.

For the continuity of $\tilde{Q}$, we write $\tilde{Q}(f, f)-\tilde{Q}(g, g)$ in terms of $f+g$ and $f-g$. By repeating all the preceding estimates, we obtain (2.6). The cases $l \neq 2$ and $k \neq 2$ follow in the same manner. This proves the main theorem completely.

\section{§3. Estimates on the Integral Operator}

Recall that the equation for the perturbation $f\left(\right.$ using $F=\mu+\mu^{1 / 2} f$ ) can 
be written as

$$
f_{i}+\hat{v} \cdot \nabla_{x} f=-\nu(v) f-K f+\tilde{Q}(f, f)
$$

where

$$
\nu(v)=\iint \frac{g \sqrt{1+g^{2}} \sigma(g, \theta)}{u_{0} v_{0}} \mu(u) d u d \Omega
$$

and

$$
\begin{aligned}
K f=\iint & \frac{g \sqrt{1+g^{2}} \sigma(g, \theta) \sqrt{\mu(u)}}{u_{0} v_{0}} \\
& \cdot\left[\sqrt{\mu\left(u^{\prime}\right)} f\left(v^{\prime}\right)+\sqrt{\mu\left(v^{\prime}\right)} f\left(u^{\prime}\right)-\sqrt{\mu(v)} f(u)\right] d u d \Omega .
\end{aligned}
$$

Here $u_{0}=\sqrt{1+|u|^{2}}, v_{0}=\sqrt{1+|v|^{2}}$. We define

$$
l=\frac{1}{2}\left(u_{0}+v_{0}\right) \text { and } j=\frac{|u \wedge v|}{2 g} .
$$

Then from [7], [9] we know that $K f=K_{2} f-K_{1} f$, where $K_{1,2}$ are integral operators

$$
K_{i} f(t, x, v)=\int_{\boldsymbol{R}^{3}} k_{i}(u, v) f(t, x, u) d u \quad(i=1,2)
$$

with the symmetric kernels

$$
\begin{aligned}
k_{1}(u, v)= & c_{1} \frac{g \sqrt{1+g^{2}} e^{-l}}{u_{0} v_{0}} \int_{0}^{\pi} \sigma(g, \theta) \sin \theta d \theta ; \\
k_{2}(u, v)= & \frac{c_{2}\left(1+g^{2}\right)^{3 / 2}}{g u_{0} v_{0}} \int_{0}^{\infty} e^{-l v \overline{1+y 2}} \sigma\left(\frac{g}{\sin (\psi / 2)}, \psi\right) \\
& \cdot \frac{y\left(1+\sqrt{\left.1+y^{2}\right)}\right.}{\sqrt{1+y^{2}}} I_{0}(j y) d y
\end{aligned}
$$

where

$$
\sin \left(\frac{\psi}{2}\right)=\frac{\sqrt{2} g}{\left[g^{2}-1+\left(g^{2}+1\right) \sqrt{1+y^{2}}\right]^{1 / 2}}
$$

and $I_{0}$ is the Bessel function of imaginary argument of order zero.

We begin by collecting several elementary inequalities.

\section{Lemma 3.1.}

$$
\begin{gathered}
\frac{\left[|u \wedge v|^{2}+|u-v|^{2}\right]^{1 / 2}}{2 u_{0}^{1 / 2} v_{0}^{1 / 2}} \leq g \leq \frac{1}{2}|u-v| \\
v_{M}=\frac{2 g \sqrt{1+g^{2}}}{u_{0} v_{0}} \leq 2 \\
l^{2}-j^{2}=\frac{g^{2}+1}{4 g^{2}}|u-v|^{2}
\end{gathered}
$$


(iv)

$$
\begin{gathered}
l^{2}-j^{2} \geq \max \left\{g^{2}+1, \frac{1}{4}|u-v|^{2}\right\} \\
\frac{1}{\sqrt{2}} g\left(1+y^{2}\right)^{1 / 4} \leq \frac{g}{\sin (\psi / 2)} \leq\left(g^{2}+1\right)^{1 / 2}\left(1+y^{2}\right)^{1 / 4} \\
\cos \frac{\psi}{2} \geq \frac{y}{2 \sqrt{1+y^{2}}} .
\end{gathered}
$$

Proof. (i) The bound $g \leq \frac{1}{2}|u-v|$ is equivalent to $4 g^{2} \leq|u-v|^{2}$, i.e. to $2 u_{0} v_{0}-2 u \cdot v-2 \leq|u-v|^{2}$, i.e. to

$$
u_{0} v_{0} \leq u \cdot v+1+\frac{1}{2}|u-v|^{2}=1+\frac{1}{2}\left(|u|^{2}+|v|^{2}\right) .
$$

Squaring both sides, we obtain the upper bound in (i). For the lower bound in (i), we write

$$
\begin{aligned}
2 g^{2} & =\frac{\left(1+|u|^{2}\right)\left(1+|v|^{2}\right)-(1+u \cdot v)^{2}}{u_{0} v_{0}+u \cdot v+1} \\
& \geq \frac{|u|^{2}+|v|^{2}+|u|^{2}|v|^{2}-2 u \cdot v-(u \cdot v)^{2}}{2 u_{0} v_{0}} \\
& =\frac{|u-v|^{2}+|u \wedge v|^{2}}{2 u_{0} v_{0}}
\end{aligned}
$$

(ii) We have

$$
2 g \sqrt{1+g^{2}}+1 \leq 2\left(g^{2}+1\right)=2\left[\frac{1}{2} u_{0} v_{0}-\frac{1}{2} u \cdot v+\frac{1}{2}\right] \leq 2 u_{0} v_{0} .
$$

(iii) From their definitions (3.3) we have

$$
\begin{aligned}
l^{2}-j^{2} & =\frac{1}{4}\left(u_{0}+v_{0}\right)^{2}-\frac{|u \wedge v|^{2}}{4 g^{2}}=\frac{1}{4}\left(\sqrt{1+|u|^{2}}+\sqrt{1+|v|^{2}}\right)^{2}-\frac{|u \wedge v|^{2}}{4 g^{2}} \\
& =\frac{g^{2}\left(2+|u|^{2}+|v|^{2}\right)+2 g^{2} \sqrt{1+|u|^{2}} \sqrt{1+|v|^{2}}|u \wedge v|^{2}}{4 g^{2}} .
\end{aligned}
$$

Thus

$$
4 g^{2}\left(l^{2}-j^{2}\right)=A-|u \wedge v|^{2}
$$

where

$$
A=g^{2}\left[2+|u|^{2}+|v|^{2}+2 \sqrt{1+|u|^{2}} \sqrt{1+|v|^{2}}\right]
$$

Using the definition of $g^{2}$, we can write $A$ as

$$
A=\frac{1}{2}\left(\sqrt{1+|u|^{2}} \sqrt{1+|v|^{2}}-u \cdot v-1\right)\left(2 \sqrt{1+|u|^{2}} \sqrt{1+|v|^{2}}+2+|u|^{2}+|v|^{2}\right)
$$




$$
\begin{aligned}
= & \frac{1}{2}\left[2\left(1+|u|^{2}\right)\left(1+|v|^{2}\right)+\sqrt{1+|u|^{2}} \sqrt{1+|v|^{2}}\left(2+|u|^{2}+|v|^{2}\right)\right. \\
& \left.-2 \sqrt{1+|u|^{2}} \sqrt{1+|v|^{2}}(u \cdot v+1)-\left(2+|u|^{2}+|v|^{2}\right)(u \cdot v+1)\right] \\
= & \frac{1}{2}\left[|u|^{2}+|v|^{2}+2|u|^{2}|v|^{2}+\sqrt{1+|u|^{2}} \sqrt{1+|v|^{2}}\left(|u|^{2}+|v|^{2}\right)\right. \\
& \left.-(u \cdot v)\left(2 \sqrt{1+|u|^{2}} \sqrt{1+|v|^{2}}+2+|u|^{2}+|v|^{2}\right)\right] \\
= & \frac{1}{2}\left[\left(|u|^{2}+|v|^{2}\right)\left(1+\sqrt{1+|u|^{2}} \sqrt{1+|v|^{2}}-u \cdot v\right)+2|u|^{2}|v|^{2}\right. \\
& \left.-2(u \cdot v)\left(\sqrt{1+|u|^{2}} \sqrt{1+|v|^{2}}+1\right)\right] \\
= & \frac{1}{2}\left[\left(|u|^{2}+|v|^{2}\right) \cdot \frac{s}{2}+2|u|^{2}|v|^{2}-2(u \cdot v)\left(\frac{s}{2}+u \cdot v\right)\right] \\
= & |u|^{2}|v|^{2}-(u \cdot v)^{2}+\frac{s}{4}\left[|u|^{2}+|v|^{2}-2 u \cdot v\right] \\
= & |u \wedge v|^{2}+\frac{s}{4}|u-v|^{2} .
\end{aligned}
$$

Hence

$$
4 g^{2}\left(l^{2}-j^{2}\right)=A-|u \wedge v|^{2}=\frac{s}{4}|u-v|^{2}=\left(g^{2}+1\right)|u-v|^{2}
$$

as claimed in (iii).

(iv) In (iii) we use $g \leq \frac{1}{2}|u-v|$ from (i) to get $l^{2}-j^{2} \geq g^{2}+1$. Combining this with (iii), we get (iv).

(v) From definition (3.7) we have

$$
\frac{g}{\sin (\psi / 2)}=\frac{\sqrt{g^{2}-1+\left(g^{2}+1\right) \sqrt{1+y^{2}}}}{\sqrt{2}}
$$

Now

$$
\begin{aligned}
g^{2}-1+\left(g^{2}+1\right) \sqrt{1+y^{2}} & \leq g^{2}+1+\left(g^{2}+1\right) \sqrt{1+y^{2}} \\
& \leq 2\left(g^{2}+1\right) \sqrt{1+y^{2}} .
\end{aligned}
$$

This gives the upper bound in (v). For the lower bound we write simply

$$
\begin{aligned}
g^{2}-1+\left(g^{2}+1\right) \sqrt{1+y^{2}} & =\left(\sqrt{1+y^{2}}-1\right)+g^{2}\left(\sqrt{1+y^{2}}+1\right) \\
& \geq g^{2}\left(\sqrt{1+y^{2}}+1\right) .
\end{aligned}
$$

(vi) The last inequality in (v) above can be restated as

$$
\frac{2 g^{2}}{\sin ^{2}(\psi / 2)} \geq g^{2}\left(\sqrt{1+y^{2}}+1\right)
$$

so that 


$$
\begin{aligned}
\cos ^{2} \frac{\psi}{2} & =1-\sin ^{2} \frac{\psi}{2} \geq 1-\frac{2}{1+\sqrt{1+y^{2}}}=\frac{\sqrt{1+y^{2}}-1}{\sqrt{1+y^{2}}+1} \\
& =\frac{y^{2}}{\left(\sqrt{1+y^{2}}+1\right)^{2}} \geq \frac{y^{2}}{4\left(1+y^{2}\right)}
\end{aligned}
$$

and this completes the proof.

Lemma 3.2. Assume (1.16)-(1.18). Then there is a positive constant $c$, depending only on $\gamma, \beta$ such that

$$
c^{-1} v_{0}^{\beta / 2} \leq \nu(v) \leq c v_{0}^{\beta / 2}
$$

for all $v \in \boldsymbol{R}^{3}$, where $v_{0}=\sqrt{1+|v|^{2}}$.

Proof. The lower bound from (1.16) gives us

$$
\nu(v) \geq c_{1} \int \frac{g^{\beta+2} \sqrt{1+g^{2}}}{(1+g) u_{0} v_{0}} e^{-v} \overline{1+|u|^{2}} \int_{0}^{\pi} \sin ^{1+\gamma} \theta d \theta .
$$

The angular integral converges and has a positive value since $r>-2$ in (1.16)(1.18). Hence

$$
\nu(v) \geq c v_{0}^{-1} \int g^{\beta+2} \frac{e^{-u_{0}}}{u_{0}} d u .
$$

By Lemma 3.1 (i), we have $g \geq \frac{|u \wedge v|}{2 u_{0}^{1 / 2} v_{0}^{1 / 2}}$; hence

$$
\begin{aligned}
\nu(v) & \geq c v_{0}^{-1} \int \frac{|u \wedge v|^{\beta+2} e^{-u_{0}}}{u_{0} \cdot u_{0}^{(\beta+2) / 2} v_{0}^{(\beta+2) / 2}} d u \\
& =c v_{0}^{-(\beta / 2)-2} \int_{0}^{\infty} \int_{0}^{\pi} \frac{\rho^{\beta+2}|v|^{\beta+2} \sin ^{\beta+2} \varphi \cdot e^{-\nu \overline{1+\rho^{2}}} \rho^{2} \sin \varphi d \rho d \varphi}{\left(1+\rho^{2}\right)^{(\beta+4) / 4}} \\
& =c v_{0}^{-(\beta / 2)-2} \cdot|v|^{\beta+2}
\end{aligned}
$$

for some constant $c>0$. For $|v|$ bounded away from the origin we have $|v| \geq c v_{0}$. This gives the lower bound of the lemma in that set. When $v=0$, we have

$$
g=g_{0} \equiv\left[\frac{\sqrt{1+|u|^{2}}-1}{2}\right]^{1 / 2}
$$

and $v_{0}=1$. Thus from the above formula we have

$$
\nu(0) \geq c \int g_{0}^{\beta+2} \frac{e^{-u_{0}}}{u_{0}} d u>0
$$

and this establishes the lower bound.

For the upper bound we write, using (1.16), 


$$
\nu(v) \leq c \int \frac{g \sqrt{g^{2}+1}}{u_{0} v_{0}} e^{-u_{0}}\left(g^{-\delta}+g^{\beta}\right) \int_{0}^{\pi} \sin ^{\gamma+1} \theta d \theta d u .
$$

The angular integral converges since $|r|<2$ if $r<0$. Recall that $0 \leq \delta<\frac{1}{2}$. By definition, $g^{2} \leq c u_{0} v_{0}$. Hence

$$
g \sqrt{g^{2}+1} \cdot g^{-\delta}=g^{1-\delta} \sqrt{g^{2}+1} \leq c\left(u_{0} v_{0}\right)^{(1-\delta) / 2}\left(1+u_{0} v_{0}\right)^{1 / 2} \leq c u_{0} v_{0} .
$$

Thus the contribution from the $g^{-\delta}$ term is bounded. As for the $g^{\beta}$ term, we estimate

$$
\int \frac{g^{\beta+1} \sqrt{1+g^{2}}}{u_{0} v_{0}} e^{-u_{0}} d u \leq c \int \frac{\left(u_{0} v_{0}\right)^{(\beta+2) / 2}}{u_{0} v_{0}} \cdot e^{-u_{0}} d u=O\left(v_{0}^{\beta / 2}\right)=O\left(|v|^{\beta / 2}\right)
$$

as desired.

In Section 4, we will need the following addition information about the function $\nu(v)$.

Lemma 3.3. $\quad \nabla_{0} \nu(v)$ is locally bounded.

Proof. By explicit computation, for $j=1,2$, or 3,

$$
\begin{aligned}
\frac{\partial}{\partial v_{j}} \nu(v)= & \frac{\partial}{\partial v_{j}}\left(v_{0}^{-1}\right) \cdot \iint \frac{g \sqrt{g^{2}+1}}{u_{0}} \sigma(g, \theta) e^{-u_{0}} d u d \Omega \\
& +\iint \frac{e^{-u_{0}}}{u_{0} v_{0}} \frac{\partial}{\partial g}\left[g \sqrt{g^{2}+1} \sigma(g, \theta)\right] \frac{\partial g}{\partial v_{j}} d u d \Omega .
\end{aligned}
$$

Since $\frac{\partial}{\partial v_{j}}\left(v_{0}^{-1}\right)=O\left(v_{0}^{-2}\right)$, the first term is $O\left(v_{0}^{(\beta / 2)-1}\right)$ by the previous lemma. For the second term, we note that by definition

$$
\begin{aligned}
4 g \frac{\partial g}{\partial v_{j}} & =\hat{v}_{j} \sqrt{1+|u|^{2}}-u_{j} \\
& =\frac{\sqrt{1+|u|^{2}}\left(v_{j}-u_{j}\right)+u_{j}\left(\sqrt{1+|u|^{2}}-\sqrt{1+|v|^{2}}\right)}{\sqrt{1+|v|^{2}}} .
\end{aligned}
$$

The function $x \mapsto \sqrt{1+|x|^{2}}$ (from $\boldsymbol{R}^{3} \rightarrow \boldsymbol{R}^{1}$ ) has Lipschitz constant bounded by 1 , hence

$$
\left|4 g \frac{\partial g}{\partial v_{j}}\right| \leq \frac{\left(\sqrt{1+|u|^{2}}+|u|\right)|u-v|}{\sqrt{1+|v|^{2}}} .
$$
We use Lemma 3.1(i) to bound $|u-v| \leq 2 u_{0}^{1 / 2} v_{0}^{1 / 2} g$. Thus $\left|\frac{\partial g}{\partial v_{j}}\right| \leq c\left(1+|u|^{2}\right)^{3 / 4}$
$\left(1+|v|^{2}\right)^{-1 / 4}$.

Next we compute the middle term in the second integral 


$$
\begin{aligned}
\left|\frac{\partial}{\partial g}\left(g \sqrt{g^{2}+1} \sigma\right)\right| & =\left|\sqrt{g^{2}+1} \sigma+\frac{g^{2} \sigma}{\sqrt{g^{2}+1}}+g \sqrt{g^{2}+1} \sigma_{g}\right| \\
& \leq 2 \sqrt{g^{2}+1} \sigma+g \sqrt{g^{2}+1}\left|\sigma_{g}\right| \\
& \leq c \sqrt{g^{2}+1}\left(g^{-\delta}+g^{\beta}\right) \sin ^{\gamma} \theta+g \sqrt{g^{2}+1}\left(g^{-\delta^{\prime}}+g^{\beta^{\prime}}\right) \sin ^{\gamma^{\prime}} \theta
\end{aligned}
$$

We need only check that this produces a locally integrable expression. Firstly, if $r$ and/or $r^{\prime}$ is negative, both $|r|$ and $\left|r^{\prime}\right|$ are less than 2 from (1.18); therefore the angular integrals converge. Those terms involving $g$ with positive exponents are easily bounded using $g \leq \frac{1}{2}|u-v|$ from Lemma 3.1 (i). The two terms which may be singular in $g$ are

$$
g^{-\delta} \text { and } g^{1-\delta^{\prime}} \text {. }
$$

If $\delta^{\prime} \leq 1$, the above reasoning applies to the second expression. We use Lemma 3.1 (i) again to write

$$
g \geq \frac{c|u-v|}{u_{0}^{1 / 2} v_{0}^{1 / 2}}
$$

Since $\delta<\frac{1}{2}$, the first term $g^{-\delta}$ is clearly locally integrable. The second $g^{1-\delta^{\prime}}$ is as well since $\delta^{\prime}<4$ by (1.17). This completes the proof.

Next we estimate the "easy" kernel $k_{1}(u, v)$ given by (3.5).

Lemma 3.4. Assume (1.16)-(1.18). Then there is a positive constant $c$ such that

$$
k_{1}(u, v) \leq \frac{c e^{-l / 2}}{|u-v|^{\delta}} .
$$

Proof. From its definition we have

$$
k_{1}(u, v)=\frac{c g \sqrt{1+g^{2}}}{u_{0} v_{0}} e^{-l} \int_{0}^{\pi} \sigma(g, \theta) \sin \theta d \theta .
$$

Applying (1.16) and noting that the angular integral is again bounded since $r>-2$, we have

$$
k_{1}(u, v) \leq c e^{-l}\left(g^{-\delta}+g^{\beta}\right)
$$

where we have used Lemma 3.1 (ii) to bound $v_{M}$. Again we use $g^{\beta} \leq\left(u_{0}^{1 / 2} v_{0}^{1 / 2}\right)^{\beta}$ $\leq c\left(u_{0}+v_{0}\right)^{\beta} \leq c l^{\beta}$. From Lemma 3.1 (i) we also have

$$
g^{-\delta} \leq c \frac{u_{0}^{\delta / 2} v_{0}^{\delta / 2}}{|u-v|^{\delta}} \leq \frac{c\left(u_{0}+v_{0}\right)^{\delta}}{|u-v|^{\delta}} \leq \frac{c l^{\delta}}{|u-v|^{\delta}} .
$$

This proves Lemma 3.4. 
Below we require estimates on special integrals involving the Bessel function $I_{0}(x)$ of imaginary argument. Here we collect several such results.

Lemma 3.5. Let $R>r \geq 0$ and consider

$$
J_{1}(R, r)=\int_{0}^{\infty} e^{-R^{2} \overline{1+x^{2}}} x I_{0}(r x) d x ; \quad J_{2}(R, r)=\int_{0}^{\infty} \frac{e^{-R^{2}} \overline{1+x^{2}} x I_{0}(r x) d x}{\sqrt{1+x^{2}}} .
$$

Then

$$
J_{1}(R, r)=\frac{R}{R^{2}-r^{2}}\left[1+\frac{1}{\sqrt{R^{2}-r^{2}}}\right] e^{-\sqrt{R^{2}-r^{2}}} ; \quad J_{2}(R, r)=\frac{e^{\sqrt{-R^{2}-r^{2}}}}{\sqrt{R^{2}-r^{2}}}
$$

Proof. With $u=\sqrt{1+x^{2}}, J_{2}$ becomes

$$
J_{2}(R, r)=\int_{1}^{\infty} e^{-R u} I_{0}\left(r \sqrt{u^{2}-1}\right) d u
$$

which is a known integral (cf. [16], p. 722, No. 2). The $J_{1}$-result follows by noting that, at least formally, $\frac{\partial J_{2}}{\partial R}=-J_{1}$.

Corollary 1. For $l, j$ defined in (3.3) and $0 \leq \zeta \leq 2$, let

$$
\tilde{I}_{\zeta}=\int_{0}^{\infty} x \exp \left(-l \sqrt{1+x^{2}}\right) I_{0}(j x)\left(1+x^{2}\right)^{-\zeta / 4} d x
$$

Then

$$
\tilde{I}_{\zeta} \leq c l^{1-\zeta / 2} e^{-|u-v| / 2} .
$$

Proof. After the identification $R=l, r=j$ we apply the Lemma. Since $l^{2}-j^{2} \geq 1$ and $l^{2}-j^{2} \geq \frac{1}{4}|u-v|^{2}$, we obtain the stated results for $\tilde{I}_{0}$ and $\widetilde{I}_{2} \mathrm{im}$ mediately. For $\tilde{I}_{\zeta}$ itself we write

$$
\begin{aligned}
\tilde{I}_{\zeta}= & \int_{0}^{\infty}\left[x^{\zeta / 2} I_{0}(j x)^{\zeta / 2} \exp \left(-l \zeta \sqrt{1+x^{2}} / 2\right)\left(1+x^{2}\right)^{-\zeta / 4}\right] \\
& \cdot\left[x^{1-\zeta / 2} I_{0}(j x)^{1-(\zeta / 2)} \exp \left\{-l[1-\zeta / 2] \sqrt{1+x^{2}}\right\}\right] d x
\end{aligned}
$$

and use the Hölder inequality with indices $p=2 / \zeta, p^{\prime}=2 /(2-\zeta)$ to get

$$
\tilde{I}_{\zeta} \leq \widetilde{I}_{\overline{2}}^{r / 2} \cdot \widetilde{I}_{0}^{(2-\zeta) / 2} \leq\left[c e^{-(1 / 2)|u-v|}\right]^{\zeta / 2}\left[c l e^{-(1 / 2)|u-v|}\right]^{1-\zeta / 2} \leq c l^{1-\zeta / 2} e^{-|u-v| / 2}
$$

as claimed.

Corollary 2. For $l$, $j$ defined in (3.3) and $0 \leq \zeta \leq 2$, let

$$
\tilde{K}_{\zeta}(l, j)=\int_{0}^{\infty} \exp \left(-l \sqrt{1+x^{2}}\right) x I_{0}(j x)\left(1+x^{2}\right)^{\zeta / 4} d x
$$

Then 


$$
\tilde{K}_{\zeta}(l, j) \leq c l^{1+\zeta / 2} e^{-|u-v| / 2}
$$

Proof. Since $\tilde{K}_{0}=\tilde{I}_{0}$, the bound is clearly true for $\zeta=0$. For $\tilde{K}_{2}$, notice that

$$
\begin{aligned}
\int_{l}^{\infty} \tilde{K}_{2}(R, j) d R & =\int_{0}^{\infty} \exp \left(-l \sqrt{1+x^{2}}\right) x I_{0}(j x) d x \\
& =\tilde{K}_{0}(l, j)=\frac{l}{l^{2}-j^{2}}\left[1+\frac{1}{\sqrt{l^{2}-j^{2}}}\right] e^{-\sqrt{l^{2}-j^{2}}}
\end{aligned}
$$

by Lemma 3.5. Differentiating with respect to $l$ and setting $R_{0}=\sqrt{l^{2}-j^{2}}$, we get

$$
\tilde{K}_{2}(l, j)=R_{0}^{-5} e^{-R_{0}}\left[\left(R_{0}^{2}+3 R_{0}+3\right) l^{2}-R_{0}^{2}-R_{0}^{3}\right]
$$

Again we use $R_{0} \geq 1$ and $R_{0} \geq \frac{1}{2}|u-v|$ from Lemma 3.1 (iv) to obtain

$$
\tilde{K}_{2}(l, j) \leq c l^{2} e^{-|u-v| / 2} .
$$

For $\tilde{K}_{\zeta}, 0<\zeta<2$ we write

$$
\tilde{K}_{\zeta}=\int_{0}^{\infty}\left[x^{\zeta / 2} e^{-(l / 2)^{\nu} \overline{1+x^{2}}} I_{0}(j x)^{\zeta / 2}\left(1+x^{2}\right)^{\zeta / 4}\right] \cdot\left[x^{1-\zeta / 2} e^{-l(1-\zeta / 2)^{\nu} \overline{1+x^{2}}} I_{0}(j x)^{1-\zeta / 2}\right] d x
$$

and apply Hölder as before with indices $p=2 / \zeta, p^{\prime}=2 /(2-\zeta)$ to obtain

$$
\widetilde{K}_{\zeta} \leq \tilde{K}_{2}^{\zeta / 2} \cdot \widetilde{K}_{0}^{1-\zeta / 2} \leq c e^{-|u-v| / 2}\left(l^{2}\right)^{\zeta / 2} \cdot(l)^{1-\zeta / 2} \leq c l^{1+\zeta / 2} e^{-|u-v| / 2}
$$

as desired.

Lemma 3.6. Let $|r|<2$ and let $l, j$ be as in (3.3). Then there is a constant $c>0$ such that

$$
I_{\gamma} \equiv \int_{0}^{1} x^{1-|\gamma|} e^{-l^{2} \sqrt{1+x^{2}}} I_{0}(j x) d x \leq c_{\gamma} e^{-c^{2} l^{2}-j^{2}} \leq c_{\gamma} e^{-(1 / 2) c|u-v|}
$$

Proof. Since $|r|<2$ the integral converges near $x=0$. Using the integral representation of the Bessel function $I_{0}(x)$ we write

$$
\begin{aligned}
I_{\gamma} & =\frac{1}{\pi} \int_{0}^{1} x^{1-|\gamma|} e^{-l^{\nu}} \overline{1+x^{2}} \int_{-1}^{1} \frac{\cosh (j x t) d t}{\sqrt{1-t^{2}}} d x \\
& \leq c \int_{-1}^{1} \frac{1}{\sqrt{1-t^{2}}}\left[\int_{0}^{1} x^{1-|\gamma|} e^{-l^{\sqrt{1+x^{2}}}+j x} d x\right] d t \\
& \leq c_{\gamma} \max _{0 \leq x \leq 1}\left[e^{-l^{2} \overline{1+x^{2}}+j_{x}}\right] .
\end{aligned}
$$

By elementary calculus, the maximum of the function

$$
g(x)=-l \sqrt{1+x^{2}}+j x
$$


occurs at $x=0,1$ or when $g^{\prime}(x)=\frac{-l x}{\sqrt{1+x^{2}}}+j=0$, i.e. when $x=x_{0}=\frac{j}{\sqrt{l^{2}-j^{2}}}$. If $x_{0} \leq 1, g(x) \leq g\left(\frac{j}{\sqrt{l^{2}-j^{2}}}\right)=-\sqrt{l^{2}-j^{2}}$ for any $x \in[0,1]$. If $x_{0}>1, g$ is increasing on $[0,1]$ and hence for $x \in[0,1]$

$$
g(x) \leq g(1)=-\sqrt{2} l+j \leq-(\sqrt{2}-1) l \leq-(\sqrt{2}-1) \sqrt{l^{2}-j^{2}}
$$

as desired.

Finally we estimate the complicated kernel $k_{2}$.

Lemma 3.7. For the kernel $k_{2}$ we have the estimate

$$
k_{2}(u, v) \leq \frac{c(1+|v|)^{[3|\gamma|+\beta+2 \delta] / 2} \cdot e^{-c|u-v|}}{\left[|u \wedge v|^{2}+|u-v|^{2}\right]^{1 / 2}|u-v|^{\delta+|\gamma|}} .
$$

provided $|\gamma|+\beta \leq 2,|\gamma|<2$ if $\gamma<0$.

Corollary。 The kernel $k_{1}$ satisfies the same bound as $k_{2}$. The Corollary follows from Lemma 3.4.

Proof. From (3.6) we have $k_{2}(u, v)=\frac{c_{2} s^{3 / 2}}{g u_{0} v_{0}} \int_{0}^{\infty} \exp \left(-l \sqrt{1+x^{2}}\right) \sigma\left(\frac{g}{\sin (\psi / 2)}, \psi\right) \frac{x\left(1+\sqrt{1+x^{2}}\right)}{\sqrt{1+x^{2}}} I_{0}(j x) d x$.

Since $s=4\left(g^{2}+1\right)$ and $g \leq \frac{1}{2}|u-v|$ by Lemma 3.1 (i), we have

$$
\frac{s^{3 / 2}}{g u_{0} v_{0}} \leq \frac{c\left[1+|u-v|^{2}\right]^{3 / 2}}{u_{0}^{1 / 2} v_{0}^{1 / 2}\left[|u \wedge v|^{2}+|u-v|^{2}\right]^{1 / 2}}
$$

Then by (1.16) we get

$$
\begin{aligned}
k_{2}(u, v) \leq & \frac{c\left[1+|u-v|^{2}\right]^{3 / 2}}{u_{0}^{1 / 2} v_{0}^{1 / 2}\left[|u \wedge v|^{2}+|u-v|^{2}\right]^{1 / 2}} \int_{0}^{\infty} x I_{0}(j x) e^{-l^{\nu} \overline{1+x^{2}}} \sin ^{\gamma} \psi \\
& \cdot\left\{g^{-\delta}\left(\sin \frac{\psi}{2}\right)^{\delta}+g^{\beta}\left(\sin \frac{\psi}{2}\right)^{-\beta}\right\} d x .
\end{aligned}
$$

Now $\sin \psi=2 \sin (\psi / 2) \cos (\psi / 2)$. If $r \geq 0$ we $\operatorname{simply}$ use $|\sin \psi| \leq 1$. If $r<0$ we use (v) and (vi) of Lemma 3.1 to write

$$
\sin \psi \geq c \frac{g}{\left(g^{2}+1\right)^{1 / 2}\left(1+x^{2}\right)^{1 / 4}} \cdot \frac{x}{\sqrt{1+x^{2}}}=\frac{\operatorname{cg} x}{\left(g^{2}+1\right)^{1 / 2}\left(1+x^{2}\right)^{3 / 4}} .
$$

Thus whatever the sign of $r$ may be, we have

$$
k_{2}(u, v) \leq \frac{c\left[1+|u-v|^{2}\right]^{3 / 2}}{u_{0}^{1 / 2} v_{0}^{1 / 2}\left[|u \wedge v|^{2}+|u-v|^{2}\right]^{1 / 2}} \int_{0}^{\infty} x I_{0}(j x) e^{-l^{2} \overline{1+x^{2}}}
$$




$$
\cdot\left\{\frac{\left(g^{2}+1\right)^{|\gamma| / 2}\left(1+x^{2}\right)^{3|\gamma| / 4}}{g^{|\gamma|} x^{|\gamma|}}\right\}\left\{g^{-\delta}\left(1+x^{2}\right)^{-\delta / 4}+\left(g^{2}+1\right)^{\beta / 2}\left(1+x^{2}\right)^{\beta / 4}\right\} d x
$$

where we have used Lemma 3.1 (v) again. We rewrite this as

$$
k_{2}(u, v) \leq \frac{c\left[1+|u-v|^{2}\right]^{3 / 2}}{u_{0}^{1 / 2} v_{0}^{1 / 2}\left[|u \wedge v|^{2}+|u-v|^{2}\right]^{1 / 2}}\left\{E_{1}+E_{2}\right\}
$$

where

$$
\begin{aligned}
& E_{1}=\frac{\left(g^{2}+1\right)^{|\gamma| / 2}}{g^{\delta+|\gamma|}} \int_{0}^{\infty} x^{1-|\gamma|} I_{0}(j x) e^{-l^{\nu}} \overline{1+x^{2}}\left(1+x^{2}\right)^{(3|\gamma|-\delta) / 4} d x ; \\
& E_{2}=\frac{\left(g^{2}+1\right)^{(\beta+|\gamma|) / 2}}{g^{|\gamma|}} \int_{0}^{\infty} x^{1-|\gamma|} I_{0}(j x) e^{-l^{2} \overline{1+x^{2}}}\left(1+x^{2}\right)^{(3|\gamma|+\beta) / 4} d x .
\end{aligned}
$$

Both of these integrals are broken at $x=1$. By Lemma 3.6, the integral over $[0,1]$ is $O\left(e^{-c|u-v|}\right)$ for some $c>0$. In the integral over $[1, \infty)$ we can replace $x^{-|\gamma|}$ by $\left(1+x^{2}\right)^{-|\gamma| / 2}$, and can then replace $\int_{1}^{\infty}(\cdots) d x$ by $\int_{0}^{\infty}(\cdots) d x$. In this manner we get

$$
\begin{aligned}
& E_{1} \leq \frac{c\left(g^{2}+1\right)^{|\gamma| / 2}}{g^{\delta+|\gamma|}}\left[e^{-c|u-v|}+\int_{0}^{\infty} x I_{0}(j x) e^{-l^{\nu} \overline{1+x^{2}}}\left(1+x^{2}\right)^{[|\gamma|-\delta] / 4} d x\right] ; \\
& E_{2} \leq \frac{c\left(g^{2}+1\right)^{(\beta+|\gamma|) / 2}}{g^{|\gamma|}}\left[e^{-c|u-v|}+\int_{0}^{\infty} x I_{0}(j x) e^{-l^{\nu} \overline{1+x^{2}}}\left(1+x^{2}\right)^{[|\gamma|+\beta] / 4} d x\right] .
\end{aligned}
$$

Since $|\gamma|+\beta \leq 2$ and $|\gamma|<2$, the first integral here is bounded by a multiple of the second, for which we have the upper bound

$$
c l^{1+(1 / 2)(|\gamma|+\beta)} e^{-|u-v| / 2}
$$

by Corollary 2 to Lemma 3.5. Hence

$$
\left|E_{1}\right|+\left|E_{2}\right| \leq \frac{c e^{-c|u-v|} l^{(|\gamma|+\beta) / 2+1}}{g^{\delta+|\gamma|}} \cdot\left[\left(g^{2}+1\right)^{|\gamma| / 2}+g^{\delta}\left(g^{2}+1\right)^{(\beta+|\gamma|) / 2}\right] .
$$

In the last factor here we use $g \leq \frac{1}{2}|u-v|$; the positive powers of $|u-v|$ which result are absorbed into the exponential. As for the denominator, we use Lemma 3.1 (i) again to get

$$
g^{\delta+|\gamma|} \geq \frac{c\left[|u \wedge v|^{2}+|u-v|^{2}\right]^{(\delta+|\gamma|) / 2}}{u_{0}^{(\delta+|\gamma|) / 2} v_{0}^{(\delta+|\gamma|) / 2}} .
$$

It follows that

$$
\begin{aligned}
\left|E_{1}\right|+\left|E_{2}\right| & \leq \frac{c\left(u_{0}^{1 / 2} v_{0}^{1 / 2}\right)^{\delta+|\gamma|} l^{(|\gamma|+\beta) / 2+1} e^{-c|u-v|}}{\left[|u \wedge v|^{2}+|u-v|^{2}\right]^{(\delta+|\gamma|) / 2}} \\
& =\frac{c l^{(3|\gamma|+\beta+2 \delta) / 2+1} \cdot e^{-c|u-v|}}{|u-v|^{\delta+|\gamma|}}
\end{aligned}
$$


Now $l=\frac{1}{2}\left(\sqrt{1+|u|^{2}}+\sqrt{1+|v|^{2}}\right)$. We write $|u| \leq|u-v|+|v|$ and replace $|u|$ by $l$ in this estimate. Then we have (with a smaller constant $c>0$ )

$$
\left|E_{1}\right|+\left|E_{2}\right| \leq \frac{c(1+|v|)^{(3|\gamma|+\beta+2 \delta) / 2} \cdot l \cdot e^{-c|u-v|}}{|u-v|^{\delta+|\gamma|}} .
$$

Using this in (3.9), we get

$$
k_{2}(u, v) \leq c\left(\frac{l}{u_{0}^{1 / 2} v_{0}^{1 / 2}}\right) \frac{(1+|v|)^{(3|\gamma|+\beta+2 \delta) / 2} \cdot e^{-c|u-v|}}{\left[|u \wedge v|^{2}+|u-v|^{2}\right]^{1 / 2}|u-v|^{\delta+|\gamma|}} .
$$

The first factor

$$
\xi \equiv \frac{l}{\sqrt{u_{0} v_{0}}}=\frac{1}{2} \frac{\left(\sqrt{1+|u|^{2}}+\sqrt{1+|v|^{2}}\right)}{\left(1+|u|^{2}\right)^{1 / 4}\left(1+|v|^{2}\right)^{1 / 4}}
$$

can be dropped for the following reason. $\xi$ is clearly bounded in the set $\frac{1}{2}|u| \leq|v| \leq 2|u|$. In the set $|v|>2|u|, \xi \leq c\left(1+|v|^{2}\right)^{1 / 4}$ and $|v-u| \geq$ $\frac{1}{2}|v|$. Thus

$$
\begin{aligned}
\xi \cdot e^{-c|u-v|} & =\left(\xi \cdot e^{-c|u-v| / 2}\right) e^{-c|u-v| / 2} \\
& \leq\left(c\left(1+|v|^{2}\right)^{1 / 4} e^{-c|v| / 4}\right) \cdot e^{-(c / 2)|u-v|} \leq c e^{-(c / 2)|u-v|}
\end{aligned}
$$

A similar argument works in the set $|v|<\frac{1}{2}|u|$. Thus we have finally proven Lemma 3.7.

Lastly we deduce several integral properties of $k(u, v)=k_{2}(u, v)-k_{1}(u, v)$ which will be used later.

Lemma 3.8. Assume (1.16)-(1.18). Then $k(u, v)$ is a symmetric kernel which satisfies

(i) $\sup _{v} \int|k(u, v)| d u<\infty$

(ii) $\sup _{v} \int k^{2}(u, v) d u<\infty$

(iii) $\int|k(u, v)|\left(1+|u|^{2}\right)^{-\alpha / 2} d u \leq c\left(1+|v|^{2}\right)^{-1 / 2(\alpha+\eta)}$

for any $\alpha \geq 0$, where

$$
\eta=1-\frac{1}{2}[3|\gamma|+\beta+2 \delta]>0 .
$$

Proof. The fact that $\eta>0$ follows from (1.18). We omit the proof of (i). We do (ii) explicitly because the singularity is much stronger in that case. Thus we have by Lemma 3.7

$$
\int k^{2}(u, v) d u \leq c(1+|v|)^{3|\gamma|+\beta+2 \delta} \int \frac{e^{-2 c|u-v|} d u}{\left[|u \wedge v|^{2}+|u-v|^{2}\right]|u-v|^{2(|\gamma|+\delta)}} .
$$


We write $u \wedge v=v \wedge(v-u)$ and set $\rho=|u-v|,|v \wedge(v-u)|=|v| \rho \sin \theta$. Then

$$
\begin{aligned}
\int k^{2}(u, v) d u & \leq c(1+|v|)^{3|\gamma|+\beta+2 \delta} \int_{0}^{\infty} \int_{0}^{\pi} \frac{\rho^{2} e^{-2 c \rho} \sin \theta d \theta d \rho}{\left[|v|^{2} \rho^{2} \sin ^{2} \theta+\rho^{2}\right] \rho^{2(|\gamma|+\delta)}} \\
& =c(1+|v|)^{3|\gamma|+\beta+2 \delta} \int_{0}^{\infty} \rho^{-2(|\gamma|+\delta)} e^{-2 c \rho} \int_{0}^{\pi} \frac{\sin \theta d \theta}{|v|^{2} \sin ^{2} \theta+1} d \rho .
\end{aligned}
$$

By explicit computation, the angular integral is $O\left(|v|^{-2} \ln |v|\right)$ for large $|v|$. The remaining $\rho$-integral converges since $|\gamma|+\delta<\frac{1}{2}$ by (1.18). Thus we have

$$
\int k^{2}(u, v) d u \leq c(\ln |v|)(1+|v|)^{3|\gamma|+\beta+2 \delta-2} .
$$

Since the exponent is negative by (1.18), (ii) follows.

Turning to (iii) we note that $k(u, v)$ decays exponentially in $|v|$ on the set $|u|<\frac{1}{2}|v|$. On the complement, using Lemma 3.7 we have

$$
\begin{aligned}
\int_{|u|>|v| / 2} \mid & k(u, v)\left|\left(1+|u|^{2}\right)^{-\alpha / 2} d u \leq c\left(1+|v|^{2}\right)^{-\alpha / 2} \int\right| k(u, v) \mid d u \\
& \leq c\left(1+|v|^{2}\right)^{-\alpha / 2}(1+|v|)^{(1 / 2)(3|\gamma|+2 \delta+\beta)} \int \frac{e^{-c|u-v|} d u}{|v \wedge(u-v)||u-v|^{\delta+|\gamma|}} \\
& \leq c\left(1+|v|^{2}\right)^{-(\alpha / 2)+(3|\gamma|+2 \delta+\beta) / 4} \int_{0}^{\infty} \int_{0}^{\pi} \frac{\rho^{2} \sin \theta e^{-c \rho} d \theta d \rho}{|v| \rho \sin \theta \cdot \rho^{|\gamma|+\delta}} \\
& \leq c\left(1+|v|^{2}\right)^{-(\alpha / 2)+(3|\gamma|+2 \delta+\beta) / 4-(1 / 2)} \\
& =c\left(1+|v|^{2}\right)^{-(1 / 2)(\alpha+\eta)}
\end{aligned}
$$

as claimed.

\section{§4. Smoothing}

In this section we prove that the linearized operator $K$ is smoothing relative to the rest of the equation. The precise definition of smoothing is as follows.

Definition ([20]). Let $A$ generate a strongly continuous semigroup $e^{-t A}$ on a Banach space $Y$. Let $K$ be a bounded linear operator: $Y \rightarrow Y$ and define

$$
U_{1}(t)=e^{-t A} K, \quad U_{n}(t)=\int_{0}^{t} U_{n-1}(s) U_{1}(t-s) d s
$$

for $n=2,3, \cdots$. We say that $K$ is $A$-smoothing if there exists an integer $l$ such that

(i) $U_{l}(t)$ is a compact operator for each $t>0$

(ii) $t \mapsto U_{l}(t)$ is continuous from $(0, \infty)$ into $\mathcal{L}(Y, Y)$ in operator norm.

Lemma 4.1. ([24]) Let $A$ and $K$ be as above and let 


$$
W\left(t_{1}, \cdots, t_{l}\right)=e^{-t_{1} A} K e^{-t_{2} A} K \cdots e^{-t_{l} A} K .
$$

If there exists a positive integer $l$ such that

(a) $W\left(t_{1}, \cdots, t_{l}\right)$ is compact for each positive $t_{1}, t_{2}, \cdots, t_{l}$, and

(b) $\left(t_{1}, \cdots, t_{l}\right) \mapsto W\left(t_{1}, \cdots, t_{l}\right)$ is a continuous function from $(0, \infty)^{l}$ into $\mathcal{L}(Y, Y)$, then $K$ is $A$-smoothing.

Proof. By definition,

$$
U_{l}(t)=\int_{0}^{t} \int_{0}^{s_{l-1}} \cdots \int_{0}^{s_{2}} W\left(s_{1}, s_{2}-s_{1}, \cdots, s_{l-1}-s_{l-2} ; t-s_{l-1}\right) d s_{1} \cdots d s_{l-1},
$$

from which the lemma easily follows.

Theorem 4.1. Let $K$ be the integral operator (1.11) and let $A=\hat{v} \cdot \nabla_{x}+\nu(v)$. Then $K$ is A-smoothing in each of the six spaces

$$
L^{2}\left(H^{k}\right), L^{2}\left(C^{k}\right), G_{\alpha}^{0}\left(H^{k}\right), G_{\alpha}^{0}\left(C^{k}\right), G_{\alpha}\left(H^{k}\right), G_{\alpha}\left(C^{k}\right)
$$

for any integer $k \geq 0$ and $\alpha>3 / 2$.

These spaces are defined in $\S 1$. We shall prove this theorem via a series of lemmas.

Lemma 4.2. If $A=\hat{v} \cdot \nabla+\nu(\bar{v})$, then $e^{-t A}$ is a bounded linear operator on each of the six spaces (4.3) with norm at most $C e^{-\nu_{0} t}$, where $\nu_{0}=\min _{v} \nu(v)$.

Proof. The operator generates the group

$$
\left(e^{-t A} f^{0}\right)(x, v)=f(t, x, v)=e^{-v(v) t} f^{0}(x-t \hat{v}, v) .
$$

Let $X=$ either $H^{k}$ or $C^{k}$. Then

$$
|f(t, \cdot, v)|_{X}=e^{-v(v) t}\left|f^{0}(\cdot, v)\right|_{X} .
$$

Therefore

$$
\begin{aligned}
\left\|f\left(t,{ }^{\circ},{ }^{\circ}\right)\right\|_{L^{2}(X)} & =\left\{\int e^{-2 \nu(v) t}\left|f^{0}(\cdot, v)\right|_{X}^{2} d v\right\}^{1 / 2} \\
& \leq e^{-v_{0} t}\left\|f^{0}\right\|_{L^{2}(X)}
\end{aligned}
$$

and

$$
\begin{aligned}
\|f(t, \cdot, \cdot)\|_{G_{\alpha}(X)} & =\sup _{v} \rho_{\alpha(}(v) e^{-v(v) t}\left|f^{0}(\cdot, v)\right|_{X} \\
& \leq e^{-v_{0} t}\left\|f^{0}\right\|_{G_{\alpha}(X)} .
\end{aligned}
$$

The conclusions follow easily.

Lemma 4.3. Let $K$ be any integral operator with a kernel which satisfies the conditions of Lemma 3.8. Then for any Banach space $X$

(a) $K: G_{\alpha}(X) \rightarrow G_{\alpha+\eta}(X)$ 
(b) $K: L^{2}(X) \rightarrow G_{0}(X) \cap L^{2}(X)$.

Proof. For (a) we have

$$
\begin{aligned}
|(K f)(v)| & \leq \int|k(u, v)|\left(1+u^{2}\right)^{-\alpha / 2}\left(1+u^{2}\right)^{\alpha / 2}|f(u)| d u \\
& \leq \int|k(u, v)|\left(1+u^{2}\right)^{-\alpha / 2} d u\|f\|_{G_{\alpha}} .
\end{aligned}
$$

Multiplying by $\left(1+v^{2}\right)^{((\alpha+\eta) / 2)}$, we deduce that

$$
\|K f\|_{G_{\alpha+\eta}} \leq c\|f\|_{G_{\alpha}} .
$$

The continuity of $K f(v)$ follows similarly.

(b) For $f \in L^{2}(X)$,

$$
|K f(v)-K f(w)| \leq\left\{\int|k(u, v)-k(u, w)|^{2} d u\right\}^{1 / 2}\|f\|_{L^{2}} .
$$

It follows that $K f(v)$ is a continuous function of $v$. Also $\|K f\|_{G_{0}}=\sup _{v}$ $|K f(v)| \leq c\|f\|_{L^{2}}$. In order to prove that $K f \in L^{2}$, we use Schwarz' inequality:

$$
|K f(v)|^{2} \leq\left\{\int|k(u, v)| d u\right\}\left\{\int|k(u, v)||f(u)|^{2} d u\right\} .
$$

Integrating over $v$ and taking the supremum of the first factor, we get

$$
\begin{aligned}
\|K f\|_{L^{2}}^{2} & \leq\left\{\sup _{v} \int|k| d u\right\} \iint|k(u, v)| d v|f(u)|^{2} d u \\
& \leq\left\{\sup _{v} \int|k| d u\right\}\left\{\sup _{u} \int|k| d v\right\}\|f\|_{L^{2}}^{2} .
\end{aligned}
$$

Lemma 4.4. Let $X$ be either $H^{k}=H^{k}(\Omega)$ or $C^{k}=C^{k}(\Omega)$. Let $p, q \in C_{c}^{\infty}\left(\boldsymbol{R}^{3}\right)$. Let $Q$ be the operator of multiplication by $q(v)$; that is, $(Q h)(x, v)=q(v) h(x)$. Let $P$ be the integral operator

$$
(P h)(x)=\int_{\boldsymbol{R}^{3}} p(v) h(x, v) d v .
$$

Then $t \mapsto P e^{-t A} Q$ is a continuous function of $t$ with values in $\mathcal{L}\left(H^{k}, H^{k+1}\right)$ and also with values in $\mathcal{L}\left(C^{k}, C^{k+1}\right)$, for each integer $k \geq 0$.

Proof. For any $h \in X$, we have

$$
\begin{aligned}
\left(P e^{-t A} Q h\right)(x) & =\int p(v) e^{-t A} q(v) h d v \\
& =\int p(v) e^{-v(v) t} q(v) h(x-t \hat{v}) d v .
\end{aligned}
$$

Let 


$$
\begin{aligned}
h_{j}(x) & =\frac{\partial}{\partial x_{j}} P e^{-t A} Q h \\
& =\int p(v) q(v) e^{-v(v) t}\left(\partial_{j} h\right)(x-t \hat{v}) d v .
\end{aligned}
$$

Let $\hat{v}=v\left(1+|v|^{2}\right)^{-1 / 2}$ or $v=\hat{v}\left(1-|\hat{v}|^{2}\right)^{-1 / 2}$. Changing variables in the integral, we have

$$
\begin{aligned}
h_{j}(x) & =\int_{|\hat{v}|<1} p q e^{-v t}\left(\partial_{j} h\right)(x-t \hat{v})\left(1-|\hat{v}|^{2}\right)^{-5 / 2} d \hat{v} \\
& =\frac{1}{t} \int_{|\hat{v}|<1} \frac{\partial}{\partial \hat{v}_{j}}\left[p q e^{-v t}\left(1-|\hat{v}|^{2}\right)^{-5 / 2}\right] h(x-t \hat{v}) d \hat{v} \\
& =\frac{1}{t} \int h(x-t \hat{v}) \sum_{i} \frac{\partial}{\partial v_{i}}\left[p q e^{-t^{\nu}}\left(1+|v|^{2}\right)^{5 / 2}\right] \frac{\partial v_{i}}{\partial \hat{v}_{j}}\left|\frac{\partial \hat{v}}{\partial v}\right| d v
\end{aligned}
$$

In this integrand appear the factors

$$
\frac{\partial v_{i}}{\partial \hat{v}_{j}}\left|\frac{\partial \hat{v}}{\partial v}\right|=\left(\delta_{i j}-v_{i} v_{j}\right)\left(1+|v|^{2}\right)^{1 / 2}\left(1+|v|^{2}\right)^{-5 / 2} .
$$

By Lemmas 3.2 and $3.3, \nu(v) \geq c|v|^{\beta / 2}$ for large $|v|$ and $\partial \nu / \partial v_{j}$ is bounded on bounded sets. Hence

$$
\left\|h_{j}\right\|_{X} \leq C(t)\|h\|_{X} \quad \text { for } \quad t \neq 0
$$

where $X=H^{k}$ or $C^{k}$ and

$$
C(t)=\frac{1}{t} \int\left|\nabla_{v}\left(p q e^{-t \nu}\left(1+|v|^{2}\right)^{5 / 2}\right)\right| \frac{d v}{1+|v|^{2}}<\infty
$$

because both $p$ and $q$ have compact supports. Inequality (4.8) leads to the conclusion.

Lemma 4.5. By a degenerate operator, we mean one of the form

$$
K=\sum_{j=1}^{N} Q_{j} P_{j}
$$

where $Q_{j}$ and $P_{j}$ are defined as in Lemma 4.4 with functions $q_{j}(v)$ and $p_{j}(v)$ in $C_{c}^{\infty}\left(\mathbb{R}^{3}\right)$, for $j=1, \cdots, N$. Then, for any positive integer $l$,

$$
J=K e^{-t_{1} A} K e^{-t_{2} A} K \cdots K e^{-t_{l} A} K
$$

is a compact mapping of $L^{2}\left(H^{k}\right)$ into $L^{2}\left(H^{k+l-\delta}\right)$ for all $\delta>0$. Also $J$ is a continuous function of $\left(t_{1}, \cdots, t_{l}\right)$ with values in that space.

Proof. We write $J$ as the finite sum

$$
J=\sum Q_{j_{1}}\left[P_{j_{1}} e^{-t_{1} A} Q_{j_{2}}\right] \cdots\left[P_{j_{l}} e^{-t_{l} A} Q_{j_{l+1}}\right] P_{j_{l+1}} .
$$


The operators in each square bracket map $H^{k}$ into $H^{k+1}$, according to Lemma 4.4. Furthermore the extra factor $P_{j_{l+1}}$ maps $L^{2}\left(H^{k}\right)$ into $H^{k}$, and the extra factor $Q_{j_{1}}$ is simply multiplication by a test function. Therefore, $J: L^{2}\left(H^{k}\right) \rightarrow$ $H_{c}^{1}\left(H^{k+l}\right)$, which is compactly embedded in $L^{2}\left(H^{k+l-\delta}\right)$.

Lemma 4.6. Let $K$ be any integral operator with a kernel which satisfies the conditions of Lemma 3.8. Then there exists a sequence of degenerate operators $K_{m}$ (in the sense of the preceding lemma) such that $\left\|K_{m}-K\right\| \rightarrow 0$ as $m \rightarrow \infty$ where \| $\|$ denotes the operator norm in $\mathcal{L}(Y, Y)$ and $Y=L^{2}(X), X$ being any Banach space.

Proof. Let $\chi_{m}(v)$ be the characteristic function of $\{|v| \leq m\}$ and let $M_{m}$ be the operator of multiplication by $\chi_{m}$. Then

$$
K=M_{m} K M_{m}+M_{m} K\left(I-M_{m}\right)+\left(I-M_{m}\right) K .
$$

The last factor is estimated as at the end of the proof of Lemma 4.3:

$$
\begin{aligned}
\left\|\left(I-M_{m}\right) K\right\|^{2} & \leq\left\{\sup _{v} \int\left(1-\chi_{m}(v)\right)|k(u, v)| d u\right\} \cdot\left\{\sup _{u} \int\left(1-\chi_{m}(v)\right)|k(u, v)| d v\right\} \\
& \leq c\left(1+m^{2}\right)^{-\eta / 2} \rightarrow 0 \text { as } m \rightarrow \infty .
\end{aligned}
$$

Similarly,

$$
\left\|M_{m} K\left(I-M_{m}\right)\right\| \leq\left\|K_{m}\left(I-M_{m}\right)\right\| \rightarrow 0 \text { as } m \rightarrow \infty .
$$

Secondly, we approximate the square integrable kernel $\chi_{m}(v) k(u, v) \chi_{m}(u)$ by a degenerate kernel $\sum_{j=1}^{N_{m}} q_{j, m}(v) p_{j, m}(v)$ where $p_{j m}$ and $q_{j m}$ belong to $L^{2}\left(\boldsymbol{R}^{3}\right)$. Finally we approximate each $p_{j m}$ and $q_{j m}$ by test functions in $C_{c}^{\infty}\left(\boldsymbol{R}^{3}\right)$. The conclusion follows easily.

Lemma 4.7. If $K$ is any operator satisfying the conditions of Lemma 3.8, then $K$ is $A$-smoothing in $L^{2}\left(H^{k}\right)$ and in $L^{2}\left(C^{k}\right)$.

Proof. By Lemma 4.6 there are degenerate operators $K_{m}$ which approximate $K$ in operator norm. For fixed $m$ we apply Lemma 4.5 with $l=1$ to deduce that $K_{m} e^{-t_{1} A} K_{m}$ is compact from $L^{2}\left(H^{k}\right)$ into $L^{2}\left(H^{k}\right)$ and is a continuous function of $t$. Therefore, by Lemma 4.2, $e^{-t_{0} A} K_{m} e^{-t_{1} A} K_{m}$ is also compact from $L^{2}\left(H^{k}\right)$ into $L^{2}\left(H^{k}\right)$ and is a continuous function of $\left(t_{0}, t_{1}\right)$ with values in $\mathcal{L}\left(L^{2}\left(H^{k}\right), L^{2}\left(H^{k}\right)\right)$. Now let $m \rightarrow \infty$. It follows from $\left\|K_{m}-K\right\| \rightarrow 0$ and Lemma 4.2 that

$$
\left\|e^{-t_{0} A} K_{m} e^{-t_{1} A} K_{m}-e^{-t_{0} A} K e^{-t_{1} A} K\right\| \rightarrow 0
$$

uniformly for $\left(t_{0}, t_{1}\right)$ belonging to any compact subset of $(0, \infty)^{2}$. Hence 
$e^{-t_{0} A} K e^{-t_{1} A} K$ is also compact and is a continuous function of $\left(t_{0}, t_{1}\right)$ with values in $\mathcal{L}\left(L^{2}\left(H^{k}\right), L^{2}\left(H^{k}\right)\right)$. By Lemma $4.1, K$ is $A$-smoothing in $L^{2}\left(H^{k}\right)$.

For the case of $L^{2}\left(C^{k}\right)$, the proof is similar. Let $K_{m} \rightarrow K$ in $\mathcal{L}\left(L^{2}\left(C^{k}\right)\right.$, $\left.L^{2}\left(C^{k}\right)\right)$. We apply Lemma 4.5 to get

$$
K_{m} e^{-t_{1} A} K_{m} \cdots K_{m} e^{-t_{l} A} K_{m}
$$

compact from $L^{2}\left(H^{k}\right)$ into $L^{2}\left(H^{k+l-\delta}\right)$, as well as continuous in the $t^{\prime}$ s. Hence

$$
e^{-t_{0} A} K_{m} e^{-t_{1} A} K_{m} \cdots K_{m} e^{-t_{l} A} K_{m}
$$

is compact and continuous from $L^{2}\left(H^{k}\right)$ into $L^{2}\left(H^{k+l-\delta}\right)$. Now $L^{2}\left(C^{k}\right) \subset L^{2}\left(H^{k}\right)$ and $L^{2}\left(H^{k+l-\delta}\right) \subset L^{2}\left(C^{k}\right)$ since the domain of the $x$ variable is bounded and we can use the Sobolev embedding with $l>3 / 2$. Therefore (4.14) is also compact and continuous from $L^{2}\left(C^{k}\right)$ into $L^{2}\left(C^{k}\right)$ by choosing $l=2$. Letting $m \rightarrow \infty$ as above, it follows that

$$
e^{-t_{0} A} K e^{-t_{1} A} K e^{-t_{2} A} K
$$

is compact from $L^{2}\left(C^{k}\right)$ into $L^{2}\left(C^{k}\right)$ and a continuous function of $\left(t_{0}, t_{1}, t_{2}\right)$. This proves that $K$ is $A$-smoothing in $L^{2}\left(C^{k}\right)$.

Proof of Theorem 4.1. The first two spaces in (4.3) have already been taken care of. Let $X$ be either $H^{k}$ or $C^{k}$. By Lemmas 4.2 and 4.3(b), the operator $e^{-r A} K$ maps $L^{2}(X) \rightarrow G_{0}(X) \rightarrow G_{0}(X)$ continuously. So by Lemmas 4.2 and 4.3(a), the operator

$$
D=e^{-r_{1} A} K e^{-r_{2} A} K \cdots e^{-r_{N} A} K e^{-r A} K
$$

maps $L^{2}(X)$ continuously into $G_{N \eta}(X)$. Hence $D$ also maps $L^{2}(X)$ into $G_{a}^{0}(X)$ for $N>\alpha / \eta$. On the other hand, by Lemma 4.7, there exists an integer $M$ such that

$$
E=e^{-s_{1} A} K e^{-s_{2} A} K \cdots e^{-s_{M} A} K
$$

is compact and continuous from $L^{2}(X)$ into $L^{2}(X)$. So the composition $D E$ is compact from $L^{2}(X)$ into $G_{\alpha}^{0}(X)$ and is a continuous function of $\left(r_{1}, \cdots, r_{N}, r\right.$, $\left.s_{1}, \cdots, s_{M}\right)$ into $\mathcal{L}\left(L^{2}(X), G_{a}^{0}(X)\right)$. Since $G_{\alpha}^{0}(X) \subset G_{\sigma}(X) \subset L^{2}(X)$ for $\alpha>3 / 2$, it follows that $D E$ has the same properties from $G_{\alpha}(X)$ into $G_{a}^{0}(X)$. Therefore $K$ is $A$-smoothing in $G_{a}(X)$ and also in $G_{a}^{0}(X)$. This completes the proof.

\section{$\S 5 . \quad$ Proof of the Main Theorem}

First we quote the abstract perturbation theorem of Vidav-Shizuta ([20], [24]). 
Theorem 5.1. Let $Y$ be a Banach space; $A, K$ as above. Assume that

(i) $\left\|e^{-t A}\right\| \leq M e^{-t \nu_{0}} \quad$ (all $t \geq 0$, some $\nu_{0}$ real)

(ii) $K$ is $A$-smoothing on $Y$. Then

(a) $A+K$ generates a strongly continuous semigroup $e^{-t(A+K)}$ such that

$$
\sigma\left(e^{-t(A+K)}\right) \cap\left\{\lambda:|\lambda|<e^{-t \nu_{0}}\right\}
$$

consists of isolated eigenvalues of finite multiplicity.

(b) $\sigma(-A-K)$ consists of a finite number of eigenvalues of finite multiplicity in each half-plane $\left\{\lambda: \operatorname{Re} \lambda>-\nu_{0}+\delta\right\}$ for every $\delta>0$. These can be labelled by

$$
\operatorname{Re} \lambda_{1} \geq \operatorname{Re} \lambda_{2} \geq \cdots \geq \operatorname{Re} \lambda_{N}>-\nu_{0}+\delta .
$$

Let $\left\{\phi_{i}\right\}_{i=1}^{N}$ denote the corresponding eigenfunctions. Assume for simplicity that these eigenvalues are simple. Then there exists a constant $c_{\delta}>0$ such that

$$
\| e^{-t(A+K)}-\sum_{j=1}^{N} e^{t \lambda_{j}}\left(\text { Proj. on } \phi_{j}\right) \|_{\mathcal{L}(Y, Y)} \leq c_{\delta} e^{\left(-\nu_{0}+\delta\right) t} .
$$

Sketch of the Proof. A sequence of operators $\left\{E_{n}(t)\right\}$ is defined recursively by

$$
\begin{gathered}
E_{1}(t)=e^{-t A} K \\
E_{n}(t)=\int_{0}^{t} E_{n-1}(s) E_{1}(t-s) d s \quad(n=2,3, \cdots) .
\end{gathered}
$$

By induction we get

$$
\left\|E_{n}(t)\right\| \leq \frac{M^{n}\|K\|^{n}}{(n-1) !} t^{n-1} e^{-\nu_{0} t}
$$

Once $-A-K$ has been shown to be a generator, two additional sequences are defined by

$$
\begin{aligned}
& H_{n}(t)=\int_{0}^{t} E_{n}(s) e^{-(t-s)(A+K)} d s, \\
& R_{n}(t)=\int_{0}^{t} E_{n}(s) H_{n}(t-s) d s .
\end{aligned}
$$

The map $t \mapsto H_{n}(t)$ is easily seen to be continuous in norm for $t \geq 0$. The operator $R_{n}(t)$ is shown to be a uniform limit of

$$
R_{n}^{\varepsilon}(t)=\int_{\varepsilon}^{t} E_{n}(s) H_{n}(t-s) d s
$$

as $\varepsilon \rightarrow 0^{+}$. Since $E_{n}(s)$ is compact by assumption, so is $R_{n}(t)$. Now set 


$$
F_{n}(t)=\int_{0}^{t} E_{n}(s) e^{-(t-s) A} d s
$$

Then using the identity

$$
e^{-t(A+K)}=e^{-t A}+\int_{0}^{t} e^{-s A} K e^{-(t-s)(A+K)} d s
$$

one gets by induction

$$
e^{-t(A+K)}=Q_{n}(t)+R_{n}(t) \quad n=1,2,3, \cdots
$$

where

$$
Q_{n}(t)=e^{-t A}+\sum_{i=1}^{2 n-1} \int_{0}^{t} E_{i}(s) e^{-(t-s) A} d s
$$

Using the above estimate for $\left\|E_{n}(t)\right\|$, we get

$$
\left\|F_{n}(t)\right\| \leq \frac{M^{n+1}\|K\|^{n}}{n !} t^{n} e^{-y_{0} t}
$$

from which it follows that

$$
\left\|Q_{n}(t)\right\| \leq e^{-v_{0} t} \cdot \sum_{j=0}^{2 n-1} \frac{M^{j+1}\|K\|^{j}}{j !} t^{j} .
$$

The key step is to apply Weyl's Theorem to conclude that the essential spectra of $Q_{n}(t)$ and $e^{-t(A+K)}$ coincide, since $R_{n}(t)$ is compact. By the bound on $Q_{n}(t)$, the spectrum of $e^{-t(A+K)}$ outside of the circle with radius $e^{-t \nu_{0}}$ must be discrete. Then, after appropriate use of the spectral mapping theorem, the result follows.

Lemma 5.1. Let $X$ be either $C^{k}$ or $H^{b}, l \geq 2$. Let $Z=\left\{f \in G_{a}^{0}(X)\right.$ : $f$ satisfies $(1.11 a)\}$. Then for every $t>0, \alpha>3 / 2$,

$$
e^{-t(A+K)}: Z \rightarrow Z \quad \text { with norm } \leq c_{\alpha} e^{-h t} \text { for some } h>0 .
$$

Proof. Let

$$
\begin{aligned}
& e^{-h t}=\max \left\{\left|e^{t \lambda_{j}}\right| \quad(j=1, \cdots, N), \quad e^{-\left(\nu_{0}-\delta\right) t}\right\} \\
& \text { i.e. } h=\min \left\{\operatorname{Re} \lambda_{j} \quad(j=1, \cdots, N), \nu_{0}-\delta\right\} \text {. }
\end{aligned}
$$

By Lemma 1.1(a), for all functions $g$ which are sufficiently small at infinity, we have

$$
\int Q^{*}(\mu, g) d v=\int v Q^{*}(\mu, g) d v=\int \sqrt{1+|v|^{2}} Q^{*}(\mu, g) d v=0 .
$$

Hence for $f$ satisfying $f_{t}+(A+K) f=0$, 


$$
\begin{aligned}
\iint \sqrt{\mu}(A+K) f d v d x & =\iint v \sqrt{\mu}(A+K) f d v d x \\
& =\iint \sqrt{1+|v|^{2}} \sqrt{\mu}(A+K) f d v d x=0 .
\end{aligned}
$$

Therefore $e^{-t(A+K)} f_{0}$ is orthogonal to the five-dimensional null space if $f_{0}$ is.

Now we convert the $L^{2}$-statement of Theorem 5.1 into an assertion in the space $Z$. It suffices to take $X=L^{2}$. We claim that, for $\alpha>3 / 2$, there is no point spectrum in the region $\operatorname{Re} \lambda \geq 0$. Indeed, if $(\lambda, g)$ were an eigenpair with $0 \neq g \in Z$, we would have

$$
\hat{v} \cdot \nabla_{x} g+\nu(v) g+K g=-\lambda g
$$

for some complex number $\lambda$. Now

$$
G_{\alpha}^{0}(X) \subset L^{2}\left(L^{2}\right) \text { whenever } \alpha>3 / 2 .
$$

Indeed, for any $H \in G_{\alpha}^{0}(X)$,

$$
\langle H, H\rangle \equiv \iint|H|^{2} d v d x=\int \rho_{\alpha}^{-2}(v)\left[\rho_{\alpha}^{2}(v) \int|H|^{2} d x\right] d v \leq c\|H\|_{\infty}^{2}
$$

since $\rho_{\alpha}^{-2} \in L^{1}$ for $\alpha>3 / 2$. Therefore we have

$$
\iint|g|^{2} d v d x<\infty
$$

i.e. $g \in G_{a}^{0} \subset L^{2}\left(L^{2}\right)$. Multiplying (5.1) by $\bar{g}$ and integrating, we get, since

$$
\begin{aligned}
& g \neq\left(a+b \cdot v+c \sqrt{1+|v|^{2}}\right) \sqrt{\mu(v)}, \\
& 0\langle\langle(\nu+K) g, g\rangle=-\operatorname{Re} \lambda \cdot\langle g, g\rangle .
\end{aligned}
$$

Here we have used Lemma 1.3 to get the strict positivity. Thus for each $j$, $1 \leq j \leq N$, we have $\operatorname{Re} \lambda_{j}<0$. Therefore $h>0$ and the proof is complete.

Lemma 5.2. Let $X=$ either $H^{k}$ or $C^{\infty}$ and write $\|\cdot\|=\|\cdot\|_{X}$. Assume (1.16) and choose any $h$ such that $\nu(v) \geq \nu_{0}>h>0$. Let $f \in C\left([0, \infty), G_{a}^{0}(X)\right)$ with the property that

$$
\|f\|_{\alpha, h} \equiv \sup _{0 \leq t<\infty} e^{h t}\|f(t)\|_{\infty}<\infty .
$$

Let $F_{\alpha, h}$ be the linear space

$$
F_{\alpha, h}=\left\{f \in C\left([0, \infty), G_{\alpha}^{0}(X)\right):\|f\|_{\alpha, h}<\infty\right\} .
$$

Then

$$
g(t)=\int_{0}^{t} \nu e^{-(t-s) A} f(s) d s
$$


belongs to $F_{\alpha, h}$ and $\|g\|_{\alpha, h} \leq c\|f\|_{\alpha, h}$ for some constant $c$.

Proof. We see that

$$
g(t, x, v)=\int_{0}^{t} e^{-v(v)(t-s)} \nu(v) f(s, x-\hat{v}(t-s), v) d s
$$

and hence

$$
\|g(t, \circ, v)\| \leq \nu(v) \int_{0}^{t} e^{-(t-s) \nu(v)}\|f(s, \cdot, v)\| d s
$$

for either choice of norm. Thus

$$
\begin{aligned}
\|g(t, \cdot, v)\| & \leq \nu(v) \cdot \sup _{s \leq t}\left\{e^{h s}\|f(s, \cdot, v)\|\right\} \cdot \int_{0}^{t} e^{-(t-s) v(v)-h s} d s \\
& \leq \frac{\nu(v)}{\nu(v)-h} \cdot e^{-h t} \cdot \sup _{s \leq t}\left\{e^{h s}\|f(s, \cdot, v)\|\right\} .
\end{aligned}
$$

Since the first factor is bounded by choice of $h$, we get

$$
\rho_{\alpha}(v)\|g(t, \circ, v)\| \leq c e^{-h t} \sup _{s \leq t}\left\{\rho_{\alpha}(v) e^{h s}\|f(s, \cdot, v)\|\right\} .
$$

Now we claim that for $f \in F_{\alpha, h}$,

$$
\rho_{\infty}(v) e^{h s}\|f(s, \cdot, v)\| \rightarrow 0 \text { as }|v| \rightarrow \infty,
$$

uniformly in $s$ for $0 \leq s \leq t$. For if not, there exists $\varepsilon_{0}>0$ and sequences $\left\{s_{n}\right\} \subset$ $\boldsymbol{R}^{1},\left\{v_{n}\right\} \subset \boldsymbol{R}^{3}$ such that $0 \leq s_{n} \leq t,\left|v_{n}\right| \rightarrow \infty$ and $H\left(s_{n}, v_{n}\right) \equiv \rho_{\infty}\left(v_{n}\right) e^{h s_{n}} \| f\left(s_{n}, \cdot\right.$, $\left.v_{n}\right) \|>\varepsilon_{0}$. By compactness we choose a subsequence $\left\{s_{n_{k}}\right\}$ for which $s_{n_{k}} \rightarrow s \in$ $[0, t]$ as $k \rightarrow \infty$. Then

$$
\begin{aligned}
\left|H\left(s_{n_{k}}, v_{n_{k}}\right)\right| & \leq\left|H\left(s_{n_{k}}, v_{n_{k}}\right)-H\left(s, v_{n_{k}}\right)\right|+\left|H\left(s, v_{n_{k}}\right)\right| \\
& \leq \sup _{v}\left|H\left(s_{n_{k}}, v\right)-H(s, v)\right|+\left|H\left(s, v_{n_{k}}\right)\right|
\end{aligned}
$$

As $k \rightarrow \infty,\left|H\left(s, v_{n_{k}}\right)\right|$ can be made arbitrarily small, because $f$, as a function of $v$, belongs to $G_{\alpha^{*}}^{0}$. For such a large choice of $k$, the first term also can be made arbitrarily small, since $H \in C\left([0, \infty), C_{0}\right)$. This contradiction proves the claim. Returning to (5.5) then and multiplying by $e^{h t}$, we see that

$$
g \in F_{\alpha, h} \text { and that }\|g\|_{\alpha, h} \leq c\|f\|_{\alpha, h} .
$$

Lemma 5.3. With the notation of Lemma 5.2 in force, let $B=A+K$ and for $0<h<\nu_{0}$ let $f \in F_{a, h}^{0} \equiv\left\{f \in C([0, \infty), Z):\|f\|_{\alpha, h}<\infty\right\}$. Set

$$
g(t)=\int_{0}^{t} e^{-(t-s) B} \nu(v) f(s) d s .
$$

Then $g \in F_{a, h}^{0}$ and $\|g\|_{\alpha, h} \leq c\|f\|_{\alpha, h}$ for some constant $c$, provided $\alpha>(3+\beta) / 2$. 
Proof. From the definition of $g$ we have

$$
\|g(t)\|_{\alpha-\beta / 2} \leq \int_{0}^{t}\left\|e^{-(t-s) B}\right\| e^{-h s} d s \cdot \max _{0 \leq s \leq t}\left\{e^{h s}\|\nu f(s)\|_{\alpha-\beta / 2}\right\} .
$$

Now choose $h_{1}$, with $0<h<h_{1}<\nu_{0}$. By Lemma 5.1, we know that

$$
\left\|e^{-t B}\right\| \leq c e^{-h_{1} t} \quad(t \geq 0)
$$

for $\alpha-(\beta / 2)>3 / 2$ and some constant $c$. Here the norm is the operator norm from $G_{\alpha-\beta / 2}^{0}(X)$ into itself. Since

$$
\int_{0}^{t} e^{-h_{1}(t-s)} e^{-h s} d s \leq \frac{e^{-h t}}{h_{1}-h}
$$

we get from the upper bound in Lemma 3.2

$$
\|g(t)\|_{\alpha-\beta / 2} \leq c e^{-h t}\|f\|_{\alpha, h} .
$$

Thus we have $g \in F_{\alpha-\beta / 2, h}$ and $\|g\|_{\alpha-\beta / 2, h} \leq c\|f\|_{\alpha, h}$ provided $\alpha>(3+\beta) / 2$.

Next we derive a simple representation for $g$. From its definition, $g(0)=0$ and

$$
g_{t}+B g=\nu f .
$$

Since $B=A+K$, this is the same as

$$
g_{t}+A g=\nu f-K g
$$

so that

$$
g(t)=\int_{0}^{t} e^{-(t-s) A} \nu f d s-\int_{0}^{t} e^{-(t-s) A} K g d s \equiv I+I I .
$$

By Lemma 5.2, $\|I\|_{\alpha, h} \leq c\|f\|_{\alpha, h}$. Term II is rewritten as

$$
I I=-\int_{0}^{t} e^{-(t-s) A} \nu\left(\nu^{-1} K g\right) d s .
$$

By the first part of the proof, $g \in F_{\alpha-\beta / 2, h}$. Using Lemma 3.8 (iii), we get

$$
K g \in F_{\alpha-\beta / 2+\eta, h}
$$

where $\eta=1-\frac{1}{2}(3|\gamma|+2 \delta+\beta)>0$ from (1.18). Then with the lower bound from Lemma 3.2 we have

$$
\nu^{-1} K g \in F_{\alpha+\eta, h} \subseteq F_{\alpha, h} .
$$

Lemma 5.2 can now be applied to term II. Lastly, from the proof of Lemma $5.1, g$ is orthogonal to the five-dimensional null space if $f$ is. 
Proof of Theorem 1.1. The perturbation from equilibrium $f$, defined in $\S 1$ by (LRB), satisfies the equation

$$
f_{t}+B f=\tilde{Q}(f, f)
$$

so that formally we have

$$
f(t)=e^{-t B} f^{0}+\int_{0}^{t} e^{-(t-\tau) B} \tilde{Q}(f, f) d \tau,
$$

where $\left.f\right|_{t=0}=f^{0}$. By Lemma 1.2(a), $\tilde{Q}$ preserves the orthogonality conditions (1.11a). Define the operator $Q f$ by

$$
Q f(t)=e^{-t B} f^{0}+\int_{0}^{t} e^{-(t-\tau) B} \tilde{Q}(f, f) d \tau .
$$

Here we take $X=$ either $H^{k}(k \geq 2)$ or $C^{m}(m \geq 0), Y=G_{a}^{0}(X), \alpha>(3+\beta) / 2, f^{0} \in Z$ and $f \in F_{a, h}^{0}$ for $0<h<\nu_{0}$. We will show that $Q f$ has a unique fixed point provided $\left\|f^{0}\right\|_{a}$ is sufficiently small.

Firstly, from Lemma 5.1, we have

$$
\left\|e^{-t B} f^{0}\right\|_{Y} \leq c e^{-h t}\left\|f^{0}\right\|_{Y} .
$$

Secondly, from Theorem 2.1, we know that for $f \in F_{\alpha, h}$ we have a constant $c$ independent of $t$ for which

$$
\|\tilde{Q}(f(t), f(t))\|_{\alpha-\beta / 2} \leq c\|f(t)\|_{\alpha}^{2}
$$

for each fixed $t \geq 0$. Applying Lemma 5.3 to

$$
\int_{0}^{t} e^{-(t-s) B} \tilde{Q}(f, f) d s=\int_{0}^{t} e^{-(t-s) B} \nu\left[\nu^{-1} \tilde{Q}(f, f)\right] d s,
$$

we get the bound

$$
\|Q f\|_{\alpha, h} \leq c_{0}\left\|f^{0}\right\|_{Y}+c_{1}\|f\|_{\alpha, h}^{2}
$$

for $\alpha>(3+\beta) / 2$.

Consider the closed ball $\mathscr{B}$ of $F_{a, h}^{0}$ given for $\delta>0$ by

$$
\mathscr{B}=\left\{f \in F_{a, h}^{0}:\|f\|_{\alpha, h} \leq \delta\right\} .
$$

We take $f^{0} \in Z$ with norm

$$
\left\|f^{0}\right\|_{Y} \leq \frac{\delta}{2 c_{0}}
$$

Then 


$$
\|Q f\|_{\infty, h} \leq \frac{\delta}{2}+c_{1} \delta^{2}
$$

Choosing $\delta \leq 1 / 2 c_{1}$ we get

$$
\|Q f\|_{\alpha, h} \leq \frac{\delta}{2}+\frac{\delta}{2}=\delta
$$

so that $Q$ maps $\mathcal{B}$ into itself for such $\delta$. From Theorem 2.1 (equation (2.3)), for $f_{1}, f_{2} \in G_{a}^{0}$ we have

$$
\left\|\tilde{Q}\left(f_{2}, f_{2}\right)-\tilde{Q}\left(f_{1}, f_{1}\right)\right\|_{\alpha-\beta / 2} \leq c\left(\left\|f_{1}\right\|_{\alpha}+\left\|f_{2}\right\|_{\alpha}\right)\left\|f_{1}-f_{2}\right\|_{\alpha} .
$$

Taking $f_{1}, f_{2} \in F_{\alpha, h}^{0}$ we compute $Q f_{1}-Q f_{2}$ and use (2.6) to estimate the nonlinear terms in (5.10). It follows that $Q$ is a contraction, so that $Q$ has a unique fixed point, and the proof is complete.

\section{Appendix I: Determination of the Maxwellian Parameters}

We are given a smooth non-negative function $F^{0}(x, v)$, periodic in $x$ and decaying in $v$. We seek 5 parameters $c>0, a \in \boldsymbol{R}$ and $b \in \boldsymbol{R}^{3}$ such that the function

$$
\mu(v) \equiv \exp \left(a+b \cdot v-c \sqrt{1+|v|^{2}}\right)
$$

satisfies

$$
0=\iint\left(F^{0}-\mu\right) d x d v=\iint v\left(F^{0}-\mu\right) d x d v=\iint \sqrt{1+|v|^{2}}\left(F^{0}-\mu\right) d x d v .
$$

We write $e^{a}=\alpha$. Then we need to solve

$$
\begin{gathered}
A=\iint F^{0} d x d v=(2 \pi)^{3} \alpha \int e^{b \cdot v-c^{2} \overline{1+||^{2}}} d v \\
B=\iint v F^{0} d x d v=(2 \pi)^{3} \alpha \int v e^{b \cdot v-c^{2} \overline{1+|v|^{2}}} d v \\
C=\iint \sqrt{1+|v|^{2}} F^{0} d x d v=(2 \pi)^{3} \alpha \int \sqrt{1+|v|^{2}} e^{b \cdot v-c^{2} \overline{1+|v|^{2}}} d v
\end{gathered}
$$

for $\alpha=e^{a}$ and $b$ and $c$.

Theorem. The nonlinear system (1)-(3) always has a solution $a, b, c$.

Proof: First we show that the relation

$$
A^{2}+|B|^{2}<C^{2}
$$

among the given parameters must hold. For this purpose, consider the measure 


$$
d \mu=\mu_{0} \exp \left(b \cdot v-c \sqrt{1+|v|^{2}}\right) d v
$$

where $b \in \mathbb{R}^{3}, c>0$ and $\mu_{0}$ is a constant for which $\int_{\mathbb{R}^{3}} d \mu=1$. Then $A^{2}+|B|^{2}<$ $C^{2}$ is the same as the inequality

$$
\left(\int d \mu\right)^{2}+\left|\int v d \mu\right|^{2}<\left(\int \sqrt{1+|v|^{2}} d \mu\right)^{2},
$$

i.e.

$$
1+\left.1 \int v d \mu\right|^{2}<\left(\int \varphi(|v|) d \mu\right)^{2}
$$

where $\varphi(s)=\sqrt{1+s^{2}}$ is strictly convex. Since $\left|\int v d \mu\right| \leq \int|v| d \mu$, it suffices to show that

$$
\sqrt{1+\left(\int|v| d \mu\right)^{2}}<\int \varphi(|v|) d \mu .
$$

This is just Jensen's inequality, and we are done. Now we show that the condition $A^{2}+|B|^{2}<C^{2}$ is sufficient for existence.

Define $I(b, c)=(2 \pi)^{3} \int e^{b \cdot v-c^{2} \sqrt{1+\mid v^{2}}} d v$. Then $I$ depends only on $|b|$ and $c$; we write $I(b, c)=\widetilde{I}(|b|, c)$ and calculate

$$
\begin{aligned}
\widetilde{I}(|b|, c) & =(2 \pi)^{3} \int_{0}^{\infty} \int_{0}^{\pi} \rho^{2} \sin \theta e^{|b| \rho \cos \theta-c^{2} \overline{1+\rho^{2}}} d \theta d \rho \\
& =\frac{2(2 \pi)^{3}}{|b|} \int_{0}^{\infty} \rho \sinh (\rho|b|) e^{-c^{2} \overline{1+\rho^{2}}} d \rho .
\end{aligned}
$$

The 3 equations can be rewritten as

$$
\begin{gathered}
A=\alpha \tilde{I}(|b|, c) \\
B=\alpha \nabla_{b} I=\alpha \frac{b}{|b|} \frac{\partial \tilde{I}}{\partial|b|} \\
C=-\alpha \frac{\partial I}{\partial c}=-\alpha \frac{\partial \tilde{I}}{\partial c} .
\end{gathered}
$$

We choose $b$ parallel to $B$. Then $\left(2^{\prime}\right)$ becomes a scalar equation, and we are reduced to 3 equations in 3 unknowns $\alpha,|b|$ and $c$. Then by $\left(2^{\prime}\right)$ we have $b \cdot B=\alpha|b| \frac{\partial \widetilde{I}}{\partial|b|}$, or

$$
\pm|B|=\alpha \frac{\partial \tilde{I}}{\partial|b|} .
$$

Now we can evaluate $\tilde{I}$ in terms of Bessel functions. For fixed $|b|$ and $c>|b|$, consider

$$
\frac{|b|}{16 \pi^{3}} \int_{c}^{\infty} \tilde{I}(b, r) d r=\int_{0}^{\infty} \rho \sinh (\rho|b|) e^{-c^{2} \overline{1+\rho^{2}}} \frac{d \rho}{\sqrt{1+\rho^{2}}}
$$




$$
\begin{aligned}
& =\int_{0}^{\infty} \rho^{3 / 2}|b|^{1 / 2} \frac{\sinh (\rho|b|)}{\rho^{1 / 2}|b|^{1 / 2}} \cdot \frac{e^{-c^{2}} \overline{1+\rho^{2}}}{c^{1 / 2}\left(1+\rho^{2}\right)^{1 / 4}} \cdot c^{1 / 2}\left(1+\rho^{2}\right)^{1 / 4} \frac{d \rho}{\sqrt{1+\rho^{2}}} \\
& =\sqrt{|b| c} \int_{0}^{\infty} \frac{\rho^{3 / 2}}{\left(1+\rho^{2}\right)^{1 / 4}} I_{1 / 2}(\rho|b|) K_{1 / 2}\left(c \sqrt{1+\rho^{2}}\right) d \rho \\
& =e^{-i(\pi / 4)} \sqrt{|b| c} \int_{0}^{\infty} \frac{\rho^{3 / 2}}{\left(1+\rho^{2}\right)^{1 / 4}} J_{1 / 2}(i \rho|b|) K_{1 / 2}\left(c \sqrt{1+\rho^{2}}\right) d \rho .
\end{aligned}
$$

If the argument of the function $J_{1 / 2}(\cdots)$ here were real, the integral would be known (cf. [16], p. 706, \#7). Under the assumption that $c>|b|$ (to be verified below at the solution) we can justify an analytic continuation to complex arguments, and get for the right-hand side

$$
\begin{aligned}
& e^{-i(\pi / 4)} \sqrt{|b| c} \cdot e^{i(\pi / 4)} \sqrt{\frac{|b|}{c}} \cdot \frac{1}{\sqrt{c^{2}-|b|^{2}}} K_{-1}\left(\sqrt{c^{2}-b^{2}}\right) \\
& =\frac{|b|}{\sqrt{c^{2}-|b|^{2}}} K_{1}\left(\sqrt{c^{2}-|b|^{2}}\right)
\end{aligned}
$$

since $K_{-\nu}=K_{\nu}$. Now call $x=\sqrt{c^{2}-|b|^{2}}$. Differentiating with respect to $c$, we get

$$
\begin{aligned}
& \frac{-|b|}{16 \pi^{3}} \tilde{I}(|b|, c)=|b| \frac{\partial}{\partial c}\left[\frac{K_{1}(x)}{x}\right] \\
& =|b| \frac{d}{d x}\left[x^{-1} K_{1}(x)\right] \frac{c}{x}=-\frac{|b| c}{x} \cdot x^{-1} K_{2}(x)
\end{aligned}
$$

and hence

$$
\widetilde{I}(|b|, c)=16 \pi^{3} \frac{c K_{2}(x)}{x^{2}}
$$

We abbreviate this by

$$
\tilde{I}(|b|, c)=c g\left(\sqrt{c^{2}-|b|^{2}}\right)
$$

with $g(y)=16 \pi^{3} y^{-2} K_{2}(y)$. Thus

$$
\begin{aligned}
& \frac{\partial \tilde{I}}{\partial|b|}=c g^{\prime}(x) \frac{d x}{d|b|}=\frac{-|b| c}{x} g^{\prime}(x) \\
& \frac{\partial \tilde{I}}{\partial c}=g(x)+c g^{\prime}(x) \frac{d x}{d c}=g(x)+\frac{c^{2}}{x} g^{\prime}(x) .
\end{aligned}
$$

We know that $\frac{d}{d x}\left(x^{-2} K_{2}(x)\right)=-x^{-2} K_{3}(x) \leq 0$. Hence $\frac{\partial \tilde{I}}{\partial|b|} \geq 0$ so that equations $\left(1^{\prime}\right)-\left(3^{\prime}\right)$ become

$$
\begin{gathered}
A=\alpha c g(x) \\
|B|=\frac{-\alpha|b| c}{x} g^{\prime}(x)
\end{gathered}
$$




$$
C=-\alpha\left(g(x)+\frac{c^{2}}{x} g^{\prime}(x)\right)
$$

Dividing the last two equations by the first, we get

$$
\begin{aligned}
\frac{|B|}{A} & =-\frac{|b|}{x} \cdot \frac{g^{\prime}(x)}{g(x)} ; \\
-\frac{C}{A} & =\frac{1}{c}+\frac{c}{x} \frac{g^{\prime}(x)}{g(x)} .
\end{aligned}
$$

Now solve (5) for $g^{\prime} / g$ and put the result into (6):

$$
-\frac{C}{A}=\frac{1}{c}+\frac{c}{x}\left[-\frac{x|B|}{|b| A}\right],
$$

so that

$$
|b|=|b(c)|=\frac{c^{2}|B|}{A+C c} .
$$

Since $A>0$ and $C \geq|B|$,

$$
|b(c)|=\frac{c^{2}|B|}{A+C c}<\frac{c^{2}|B|}{C c} \leq c .
$$

Thus any sloution $c$ generates a solution $|b|=|b(c)|$ for which the condition $|b|<c$ holds.

It remains to solve for $c$. By recursions for the Bessel functions,

$$
\frac{g^{\prime}(x)}{g(x)}=\frac{\left(x^{-2} K_{2}(x)\right)^{\prime}}{x^{-2} K_{2}(x)}=-\frac{x^{-2} K_{3}(x)}{x^{-2} K_{2}(x)}=-\frac{K_{3}(x)}{K_{2}(x)} .
$$

Therefore (6) can be rewritten as

$$
\frac{x K_{2}(x)}{K_{3}(x)}=\frac{A c^{2}}{A+C c} .
$$

Since $|b|=|b(c)|$ is known from (7), (8) is an equation in $c$ alone. In fact, by definition and (7),

$$
x^{2} \equiv c^{2}-|b|^{2}=c^{2}-\frac{c^{4}|B|^{2}}{(A+c C)^{2}}
$$

so that

$$
x=x(c)=\frac{c}{A+C c} \sqrt{(A+C c)^{2}-|B|^{2} c^{2}} .
$$

Now consider (8) rewritten as $q(c)=0$, where

$$
q(c)=\frac{A c^{2}}{A+C c}-\frac{x(c) K_{2}(x(c))}{K_{3}(x(c))}
$$


From (9) we see that

$$
\lim _{c \rightarrow 0^{+}} \frac{x(c)}{c}=1, \lim _{c \rightarrow \infty} \frac{x(c)}{c}=\frac{\sqrt{C^{2}-|B|^{2}}}{C} .
$$

From the series definitions of the Bessel functions, we have near $y=0$,

$$
\frac{y K_{2}(y)}{K_{3}(y)} \sim \frac{y \cdot \frac{1}{2}\left(\frac{y}{2}\right)^{-2}}{\left(\frac{y}{2}\right)^{-3}}=\left(\frac{y}{2}\right)^{2} .
$$

Thus as $c \rightarrow 0^{+}$,

$$
q(c) \sim c^{2}-\frac{1}{4} x(c)^{2} \sim \frac{3}{4} c^{2}
$$

and therefore $q(c)>0$ for small $c>0$.

For large arguments, both $K_{2}(y)$ and $K_{3}(y)$ have the asymptotic representations $\sqrt{\pi / 2 y} e^{-y}$. As $c \rightarrow \infty$ then,

$$
q(c) \sim \frac{A}{C} c-x(c) \sim c\left[\frac{A}{C}-\frac{\sqrt{C^{2}-|B|^{2}}}{C}\right] .
$$

As was shown at the beginning of the proof, this is negative. Since $q$ is continuous in $\{c>0\}$, we are done.

\section{Appendix II: The Collision Integral}

We derive an alternative form of the collision integral which is closer to the classical form. We shall also prove Lemma 1.1. We begin by carrying out the first three delta functions in (0.2) to get

$$
\begin{aligned}
Q(F, F)(v)= & \frac{1}{2 v_{0}} \iiint \delta^{(4)}\left(U+V-U^{\prime}-V^{\prime}\right) \\
& \cdot S \sigma\left[F\left(u^{\prime}\right) F\left(v^{\prime}\right)-F(u) F(v)\right] \frac{d^{3} u}{u_{0}} \frac{d^{3} u^{\prime}}{u_{0}^{\prime}} \frac{d^{3} v^{\prime}}{v_{0}^{\prime}}
\end{aligned}
$$

where $u_{0}=\sqrt{1+|u|^{2}}$, etc. Next, we work out three more delta functions to get

$$
\begin{aligned}
Q(F, F)(v)= & \frac{1}{2} \iint \frac{s \sigma}{u_{0} v_{0} u_{0}^{\prime} v_{0}^{\prime}} \delta\left(u_{0}+v_{0}-u_{0}^{\prime}-v_{0}^{\prime}\right) \\
& \cdot\left[F\left(u^{\prime}\right) F\left(v^{\prime}\right)-F(u) F(v)\right] d^{3} v^{\prime} d^{3} u .
\end{aligned}
$$

Because of (0.3) we may write

$$
v^{\prime}=v-r \omega, u^{\prime}=u+r \omega
$$


where $\omega \in S^{2}$ and $r \in[0, \infty)$ are spherical coordinates. Then

$$
A=\frac{1}{u_{0}^{\prime} v_{0}^{\prime}} \delta\left(u_{0}^{\prime}+v_{0}^{\prime}-u_{0}-v_{0}\right) d^{3} v^{\prime}=\frac{2\left(u_{0}+v_{0}\right)}{u_{0}^{\prime} v_{0}^{\prime}} \delta\left(\left(u_{0}^{\prime}+v_{0}^{\prime}\right)^{2}-\left(u_{0}+v_{0}\right)^{2}\right) d^{3} v^{\prime}
$$

using the identity $\delta(\lambda-a)=2 a \delta\left(\lambda^{2}-a^{2}\right)$ for $\lambda>0, a>0$. Noting that

$$
\left(u_{0}^{\prime}+v_{0}^{\prime}\right)^{2}-\left(u_{0}+v_{0}\right)^{2}=2 u_{0}^{\prime} v_{0}^{\prime}+u_{0}^{\prime 2}+v_{0}^{\prime 2}-\left(u_{0}+v_{0}\right)^{2}
$$

and using the identity again, we have

$$
A=\frac{2\left(u_{0}+v_{0}\right)}{u_{0}^{\prime} v_{0}^{\prime}} 2 u_{0}^{\prime} v_{0}^{\prime} \delta\left(4 u_{0}^{\prime 2} v_{0}^{\prime 2}-\left[\left(u_{0}+v_{0}\right)^{2}-u_{0}^{\prime 2}-v_{0}^{\prime 2}\right]^{2}\right) d^{3} v^{\prime}
$$

Using the coordinates above, we see that the argument in this delta function is

$$
p(r)=-\left(u_{0}+v_{0}\right)^{4}+2\left(u_{0}+v_{0}\right)^{2}\left(u_{0}^{\prime 2}+v_{0}^{\prime 2}\right)-\left(u_{0}^{\prime 2}-v_{0}^{\prime 2}\right)^{2},
$$

which is a polynomial of degree $\leq 4$. Because

$$
u_{0}^{\prime 2}-v_{0}^{\prime 2}=|u+r \omega|^{2}-|v-r \omega|^{2}=|u|^{2}-|v|^{2}+2 r(u+v) \cdot \omega,
$$

it is merely a quadratic. It is also easy to see that $r=0$ is one of its roots. The other one is denoted by $a=a(u, v, \omega)$. Thus $p(r)$ has the form

$$
p(r)=D r^{2}-2 N r, a=2 N / D \text {. }
$$

It follows that

$$
\begin{aligned}
A & =4\left(u_{0}+v_{0}\right) \delta(D r(r-a)) r^{2} d r d \omega \\
& =4\left(u_{0}+v_{0}\right)|D a|^{-1}[\delta(r)+\delta(r-a)] r^{2} d r d \omega .
\end{aligned}
$$

Here we have used the identity for $a \neq b$

$$
\delta((\lambda-a)(\lambda-b))=\frac{1}{|a-b|}[\delta(\lambda-a)+\delta(\lambda-b)] .
$$

The first delta function drops out because of the factor $r^{2}$. Thus, putting $r=a=2 N / D$,

$$
A=4\left(u_{0}+v_{0}\right) 2|N| D^{-2} \delta(r-a) d r d \omega
$$

From [13],

$$
\begin{aligned}
& N=\left(u_{0}+v_{0}\right) u_{0} v_{0} \omega \cdot(\hat{v}-\hat{u}), \quad \text { and } \\
& D=\left(u_{0}+v_{0}\right)^{2}-(\omega \cdot(u+v))^{2} .
\end{aligned}
$$

Hence

$$
A=8 \frac{\left(u_{0}+v_{0}\right)^{2} u_{0} v_{0}|\omega \cdot(\hat{v}-\hat{u})|}{\left[\left(u_{0}+v_{0}\right)^{2}-(\omega \cdot(u+v))^{2}\right]^{2}} \delta(r-a) d r d \omega
$$


Thus

$$
\begin{aligned}
Q(F, F)(v) & =4 \iint \frac{s \sigma}{u_{0} v_{0}} \frac{\left(u_{0}+v_{0}\right)^{2} u_{0} v_{0}|\omega \cdot(\hat{v}-\hat{u})|}{\left[\left(u_{0}+v_{0}\right)^{2}-(\omega \cdot(u+v))^{2}\right]^{2}}\left(F^{\prime} F^{\prime}-F F\right) d^{2} \omega d^{3} u \\
& =\iint q(u, v, \omega)\left[F\left(u^{\prime}\right) F\left(v^{\prime}\right)-F(u) F(v)\right] d \omega d u
\end{aligned}
$$

where

$$
q(u, v, \omega)=4 s \sigma \frac{\left(u_{0}+v_{0}\right)^{2}|\omega \cdot(\hat{v}-\hat{u})|}{\left[\left(u_{0}+v_{0}\right)^{2}-(\omega \cdot(u+v))^{2}\right]^{2}}
$$

This kernel has the properties

$$
0 \leq q(u, v, \omega) \leq s \sigma\left(u_{0}+v_{0}\right)^{2}|\omega \cdot(\hat{v}-\hat{u})|
$$

because $D \geq 2$, and

$$
q(u, v, \omega)=q(v, u, \omega)
$$

In the classical limit

$$
q(u, v, \omega) \sim 4 s \sigma \frac{(1+1)^{2}|\omega \cdot(v-u)|}{\left[(1+1)^{2}-0^{2}\right]^{2}}=4 s \sigma|\omega \cdot(v-u)| .
$$

Proof of Lemma 1.1. We must prove that (1.5) implies (1.6). In view of the above calculation, we may replace $v_{M} \sigma d \Omega$ by $q d \omega$ in both (1.5) and (1.6). Now the first of the four identities (1.6), with $\varphi(v)$, is equivalent to the definition (1.5). Next we switch $u$ and $v$. Then

$$
u^{\prime}=u+a(u, v, \omega) \omega \rightarrow v+a(v, u, \omega) \omega=v-a(u, v, \omega) \omega=v^{\prime}
$$

and similarly $v^{\prime} \rightarrow u^{\prime}$, because $a(u, v, \omega)$ is antisymmetric in $u$ and $v$. So

$$
\begin{aligned}
2 \int Q^{*}(f, g) \varphi d v= & \iiint q(v, u, \omega)\left[f\left(u^{\prime}\right) g\left(v^{\prime}\right)+f\left(v^{\prime}\right) g\left(u^{\prime}\right)\right. \\
& -f(u) g(v)-f(v) g(u)] \varphi(u) d u d v d \omega,
\end{aligned}
$$

which proves the second of the identities in (1.6). Next, we change variables $(u, v) \rightarrow\left(u^{\prime}, v^{\prime}\right)$ in the last identity:

$2 \int Q^{*}(f, g) \varphi d v=\iiint q(u, v, \omega)\left\lfloor f\left(u^{\prime}\right) g\left(v^{\prime}\right)+\cdots\right] \varphi(u)\left|\frac{\partial(u, v)}{\partial\left(u^{\prime}, v^{\prime}\right)}\right| d u^{\prime} d v^{\prime} d \omega$.

On the right side we rename $(u, v) \leftrightarrow\left(u^{\prime}, v^{\prime}\right)$ to get

$$
\begin{gathered}
\iiint q\left(u^{\prime}, v^{\prime}, \omega\right)\left[f^{\prime}(u) g(v)+\cdots\right] \varphi\left(u^{\prime}\right)\left|\frac{\partial\left(u^{\prime}, v^{\prime}\right)}{\partial(u, v)}\right| d u d v d \omega \\
=\iiint q(u, v, \omega)[f(u) g(v)+\cdots] \varphi\left(u^{\prime}\right) d u d v d \omega
\end{gathered}
$$


where we have used the property

$$
q(u, v, \omega)=q\left(u^{\prime}, v^{\prime}, \omega\right) \frac{\partial\left(u^{\prime}, v^{\prime}\right)}{\partial(u, v)}
$$

or

$$
u_{0} v_{0} q(u, v, \omega)=u_{0}^{\prime} v_{0}^{\prime} q\left(u^{\prime}, v^{\prime}, \omega\right)
$$

or

$$
\frac{|N(u, v, \omega)|}{[D(u, v, \omega)]^{2}}=\frac{\left|N\left(u^{\prime}, v^{\prime}, \omega\right)\right|}{\left[D\left(u^{\prime}, v^{\prime}, \omega\right)\right]^{2}} .
$$

Since $D(u, v, \omega)=D\left(u^{\prime}, v^{\prime}, \omega\right)$ and $a(u, v, \omega)=-a\left(u^{\prime}, v^{\prime}, \omega\right)$, the last equation is true. This proves the fourth identity in (1.6). We get the third identity in (1.6) by switching $u$ and $v$.

\section{References}

[1] Bardos, C., Degond, P. and Golse F., A priori estimates and existence results for the Vlasov and Boltzmann Equations, in: Proc. 1984 AMS-SIAM Summer Seminar, Santa Fe.

[2] Bellomo, N., Lachowicz, M., Palczewski, A. and Toscani, G., On the Initial Value Problem for the Boltzmann Equation with a force term, Trans. Th. Stat. Phys., 18 (1989), 87-102.

[3] Caflisch, R., The Boltzmann Equation with Soft Potentials, Comm. Math. Phys., 74 (1980), 71-109.

[4] Carleman, T., Problèmes Mathématiques dans la Théorie Cinétique des Gaz, Almquist et Wiksell, Uppsala, 1957, and Acta Math., 60 (1933), 91.

[5] Cercignani, C., The Boltzmann Equation and its Applications, Springer-Verlag, New York, 1988.

[6] Ceregnani, C. and Majorana, A., Analysis of thermal, sound, and shear waves according to a relativistic kinetic model, Phys. Fluids, 28 (1985), 1673-1683.

[7] de Groot, S.R., van Leeuwen, W.A. and van Weert, C.G., Relativistic Kinetic Theory, North-Holland, Amsterdam, 1980.

[8] DiPerna, R. and Lions, P-L., On the Cauchy Problem for Boltzmann Equations: Global Existence and Weak Stability, Ann. Math., 130 (1989), 321-366.

[9] Dudyński, M. and Ekiel-Jezewska, M., On the Linearized Relativistic Boltzmann Equation, Comm. Math. Phys., 115 (1988), 607-629.

[10] Glassey, R. and Strauss, W., Singularity formation in a collisionless plasma could only occur at high velocities, Arch. Rat. Mech. Anal., 92 (1986), 56-90.

[11] - High velocity particles in a collisionless plasma, Math. Meth. Appl. Sci., 9 (1987), 46-52.

[12] - Absence of shocks in an initially dilute collisionless plasma, Comm. Math. Phys., 113 (198\%), 191-208.

[13] - On the Derivatives of the Collision Map of Relativistic Particles, Trans. Th. Stat. Phys., 20 (1991), 55-68.

[14] Golse, F., Lions, P-L., Perthame, B. and Sentis, R., Regularity of the Moments of the solution to a Transport Equation, J. Funct. Anal., 76 (1988), 110-125. 
[15] Grad, H., Asymptotic Theory of the Boltzmann Equation, II. in: Rarefied Gas Dynamics, 1, J. Laurmann, ed. Academic Press, N.Y. (1963).

[16] Gradshteyn, I. and Ryzhik, I., Tables of Integrals, Series and Products, Academic Press, New York, 1965.

[17] Illner, R. and Shinbrot, M., The Boltzmann Equation, global existence for a rare gas in an infinite vacuum, Comm. Math. Phys., 95 (1984), 217-226.

[18] Kaniel, S. and Shinbrot, M., The Boltzmann Equation, uniqueness and local existence, Comm. Math. Phys., 58 (1978), 65-84.

[19] Nishida, T. and Imai, K., Global Solutions to the initial value problem for the nonlinear Boltzmann Equation, Publ. RIMS Kyoto Univ., 12 (1976), 229-239.

[20] Shizuta, Y., On the Classical Solutions of the Boltzmann Equation, Comm. Pure Appl. Math., 36 (1983), 705-754.

[21] Stewart, John M., Non-Equilibrium Relativistic Kinetic Thcory. Lecture Notes in Physics 10, Springer-Verlag, 1971.

[22] Ukai, S., Solutions of the Boltzmann Equation, Studies in Math. Appl., 18 (1986), $37-$ 96.

[23] — On the Existence of Global Solutions of a mixed problem for the nonlinear Boltzmann equation, Proc. Japan. Acad., 50 (1974), 179-184.

[24] Vidav, I., Spectra of perturbed semigroups with applications to transport theory, $J$. Math. Anal. Appl., 30 (1970), 264-279. 
